\title{
Iterated Transformation-Kernel Density Estimation
}

\author{
Lijian YANG and James S. MARRON*
}

December, 1996

\begin{abstract}
Transformation from a parametric family can improve the performance of kernel density estimation. In this paper, we give two data-driven estimators for the optimal transformation parameter. We demonstrate that multiple families of transformations can be employed at the same time, and there can be benefits to iterating this process. The transformation scheme can be expected to first pick the right transformation family and then the optimal parameter. Insight as to the performance of the method comes from our analysis of a number of real datasets, two of which are included in this paper. To illustrate the effectiveness and asymptotics of the transformation method, we also present results on one of the five target densities used in our simulation study. It is then proved that the Johnson Family of transformations, when coupled with transformation-kernel density estimation, makes a wide variety of density shapes easier to estimate. The transformation method has overall better performance than the usual method and in many cases it is much more effective.
\end{abstract}

KEY WORDS: Global bandwidth; Johnson Family; Kernel estimation; Normal mixture; Pilot estimator; Transformation.

\section{INTRODUCTION}

Nonparametric density estimation extracts information about the underlying structure of a dataset

*Lijian Yang is Postdoctoral Fellow, Statistics Department, University of North Carolina, Chapel Hill, North Carolina 27599. James S. Marron is Professor, Statistics Department, University of North Carolina, Chapel Hill, North Carolina 27599. The results presented here are part of Lijian Yang's Ph.D. work under the direction of James Marron. We want to thank J. Fan, C. Ji, D. Ruppert, J. S. Simonoff, Y. Truong, an associate editor and an anonymous referee for their many useful suggestions. The revised version was finished while Lijian Yang was visiting Humboldt University in Berlin and financially supported by Sonderforschungsbereich 373 "Quantifikation und Simulation Ökonomischer Prozesse" Deutsche Forschungsgemeinschaft. 
when no appropriate parametric model is available. In estimating the density function $f_{X}$ of an i.i.d. random sample $X_{1}, \ldots, X_{n}$, the kernel density estimator (KDE) is easy to understand intuitively, it puts 'bumps' of the same shape at every observation and averages them

$$
\widehat{f}_{X}(x)=n^{-1} \sum_{i=1}^{n} K_{h}\left(x-X_{i}\right)
$$

where $K_{h}(u)=h^{-1} K(u / h)$. The kernel $K$ is a symmetric probability density, $h>0$ is the bandwidth.

Very often, when the true density has sharp features such as high skewness or kurtosis, the KDE using a global bandwidth either obscures these important features or creates extra features as shown in Figure 1. The data consists of the numbers of international adoption visas granted to U.S. residents by the Immigration and Naturalization Service in 1991, by the country of origin of the adoptee. Only countries with nonzero values are included, see Chatterjee, Handcock, and Simonoff (1995), page 287.

Figure 1 shows that the kernel estimates with smaller bandwidth (i.e., $\frac{1}{5} h_{S J}$ ) captures the peak on the left, yet is too wiggly; the one with larger bandwidth (i.e., $5 h_{S J}$ ) is smooth, yet smooths away the peak; the one using the Sheather-Jones automatic bandwidth $\left(h_{S . J}\right)$ is an attempt to balance the needs to capture the peak and to remain smooth. In the sense of overall performance, the Sheather-Jones bandwidth is a very popular global bandwidth, but no global bandwidth can produce a satisfactory density estimate for the Adoption Visa data.

We have developed an algorithm that transforms a dataset first and then uses the Sheather-Jones bandwidth to estimate the density of the transformed data set. That density estimate is then backtransformed to the original scale to estimate the original density. This can be iterated any number of times. We find transforming the data twice yields an estimate much superior to the estimate without transformation, and in most cases, little improvement is achieved after two transformation steps. So here we study the estimates obtained by transforming the original data once and twice. Figure 2 illustrates the improvements thus obtained.

Figure 2 shows these two estimates overlaid with the estimate not using transformation with bandwidth $h_{S J}$ from Figure 1. The data set used is the same Adoption Visa data. The Transformed estimate is better than the Untransformed in overall smoothness and capturing the left peak. Similarly, the Transformed Twice estimate is better than the Transformed estimate in both respects.

The Transformed estimate works like a variable bandwidth estimate: it estimates the density at the left peak as if using a small global bandwidth (e.g., $\frac{1}{5} h_{S J}$ ), while using a large global bandwidth (e.g., $5 h_{S J}$ ) toward the right tail. The Transformed Twice method does the same thing, only the effect is stronger. The advantage of using transformation is that it still allows the use of a global 
bandwidth, albeit on a transformed scale. The different amount of smoothing needed at different locations is absorbed in the transformation, making it possible to use a global bandwidth effectively. This is important since much is known about global bandwidth choice [see Jones, Marron and Sheather (1992)], but not for local choice. For references on the transformation method, see Devroye and Györfi (1985), Silverman (1986). Related recent works include: Wand, Marron and Ruppert (1991), Park, Chung and Seog (1992), Ruppert and Wand (1992), Ruppert and Cline (1994), Yang (1995b), and the dissertation of Yang (1995a).

In Section 2 we present the setting of transformation in density estimation, examine its functional analysis, and introduce a data-driven method for implementation. In particular, the use of Johnson Family is discussed. We then show that the method is sensitive enough to select the best family of transformations for a given density. In Section 3 we give two real data examples. In Section 4 we present key simulation results. In Section 5, we give a sufficient condition for an effective transformation and also prove that the Johnson Family of transformations can improve the estimation of all density shapes that are $C^{3}(R)$ (we use the notation $C^{k}(R)$ for the space of functions with continuous $k$-th derivative on $R$.) and rapidly decreasing. Our conclusions are contained in Section 6 and technical proofs are in the Appendix.

\section{THE THEORY AND METHOD}

Wand, Marron and Ruppert (1991) proposed selection of a transformation from a parametric family $\left\{g_{\lambda}\right\}$, where $\lambda \in \Lambda$, is an interval of finite dimension. Each $g_{\lambda}$ is a transformation well-defined on $S\left(f_{X}\right)$, the support of $f_{X}$, and each transforms the density $f_{X}$ to a density

$$
f_{Y}(y, \lambda)=f_{X}\left\{g_{\lambda}^{-1}(y)\right\}\left(g_{\lambda}^{-1}\right)^{\prime}(y)
$$

From the family $\left\{f_{Y}(y, \lambda)\right\}$ of densities, one for each value of the parameter $\lambda$, we would select the one easiest to estimate with a global bandwidth. Suppose that it is $f_{Y}\left(y, \lambda_{0}\right)$. We need a functional $G(\cdot)$ of density functions such that $f_{Y}\left(\cdot, \lambda_{0}\right)$ has the least $G(\cdot)$-values among the $f_{Y}(y, \lambda)$ 's. This functional $G(\cdot)$ measures the difficulty of estimation with a global bandwidth. It should be scale-invariant since every density remains as easy to estimate under rescaling. Two such functionals were considered in Wand and Devroye (1993), each measures how well the kernel estimator converges to the true density in the $L^{2}$ and $L^{1}$ norms respectively. We use the one for $L^{2}$-theory, for convenience of obtaining asymptotic results and implementing the algorithm. We now describe this functional in context. 
The mean integrated squared error (MISE) of estimating $f_{X}$ with the kernel estimator $\hat{f}_{X}$ in (1.1) is

$$
\operatorname{MISE}(h)=E \int\left\{\hat{f}_{X}(x)-f_{X}(x)\right\}^{2} d x .
$$

As $n \rightarrow \infty, h \rightarrow 0$, and $n h \rightarrow \infty$, if $f_{X}$ has a uniformly continuous second derivative, we have

$$
\operatorname{MISE}(h)=\operatorname{AMISE}(h)+o\left(\frac{1}{n h}+h^{4}\right),
$$

where

$$
A M I S E(h)=\frac{R(K)}{n h}+\frac{1}{4} \sigma_{K}^{4} h^{4} R\left(f_{X}^{\prime \prime}\right)
$$

is the asymptotic mean integrated squared error which is approximately the same as MISE, with $R(\psi)=\int(\psi)^{2}$ for any function $\psi$ and $\sigma_{K}^{2}=\int x^{2} K(x) d x$ [Jones, Marron and Sheather (1992)]. The AMISE is minimized by the following asymptotically optimal bandwidth

$$
h_{*}=\left(\frac{R(K)}{\sigma_{K}^{4} R\left(f_{X}^{\prime \prime}\right)}\right)^{\frac{1}{5}} n^{-\frac{1}{5}} .
$$

Plugging this $h_{*}$ back into (2.4), we have

$$
\inf _{h>0} A M I S E(h)=C(K) n^{-4 / 5} R\left(f_{X}^{\prime \prime}\right)^{\frac{1}{5}}=C(K) n^{-4 / 5}\left[\int\left[f_{X}^{\prime \prime}(x)\right]^{2} d x\right]^{1 / 5},
$$

where $C(K)$ is a constant depending on $K$ only. It is clear from (2.6) that when $C(K)$ is fixed, smaller values of $R\left(f_{X}^{\prime \prime}\right)$ yields smaller $L^{2}$ errors of estimating $f_{X}$ with $\hat{f}_{X}$. Therefore, it is "easier" to estimate $f_{X}$ when $R\left(f_{X}^{\prime \prime}\right)$ is smaller. Another way of looking at this is from the fact that the global asymptotic optimal bandwidth $h_{*}$ in $(2.5)$ is larger when $R\left(f_{X}^{\prime \prime}\right)$ is smaller; in general a smaller optimal bandwidth is needed when the underlying density is wiggly. Finally, geometrically speaking, $R\left(f_{X}^{\prime \prime}\right)$ is a global measure of the curvature of the density, and less curvature makes estimation easier.

Hence $R\left(f_{X}^{\prime \prime}\right)$ is a functional measuring how easy it is to estimate $f_{X}$. Since $R\left(\left[\frac{1}{c} f_{X}(\dot{c})\right]^{\prime \prime}\right)=$ $\frac{1}{c^{5}} R\left(f_{X}^{\prime \prime}\right)$, a scale invariant version of $R\left(f_{X}^{\prime \prime}\right)$ is

$$
G\left(f_{X}\right)=\sigma\left(f_{X}\right)\left(R\left(f_{X}^{\prime \prime}\right)\right)^{1 / 5}=\sigma_{f_{X}}\left(R\left(f_{X}^{\prime \prime}\right)\right)^{1 / 5}
$$

where $\sigma_{f_{X}}^{2}=\sigma\left(f_{X}\right)^{2}$ is the variance of the distribution whose density is $f_{X}$. One can verify that $G\left(f_{X}\right)$ is invariant under rescaling. The smaller $G\left(f_{X}\right)$ is, the larger the scale free optimal bandwidth $h_{*}$ is, thus the easier the estimation.

Terrell (1990) proved the following general result: 
Among those $C^{k}(R)$ densities defined on the real line with specified variance $\sigma^{2}$, the member of the scale family of beta $(k+2, k+2)$ has the smallest value of $\int\left(f^{(k)}\right)^{2}$, i.e., the density that achieves the minimum is a rescaling of the function

$$
f(x)=\frac{(2 k+3) !}{2^{2 k+3}((k+1) !)^{2}}\left(1-x^{2}\right)^{k+1} 1_{\{|x| \leq 1\}}
$$

If $k=2$, Terrell's result says in particular that $G(\cdot)$ is minimized by beta(4,4). The minimum is $\left(\frac{35}{243}\right)^{1 / 5} \approx 0.6787$. The normal density is close to attaining this lower bound value $\left(=\left(\frac{3}{8 \sqrt{\pi}}\right)^{1 / 5} \approx\right.$ $0.733)$, and is similar to the beta $(4,4)$ in shape.

Our transformation-kernel density estimation (TKDE) method attempts to reduce $G(\cdot)$ as much as possible by transforming $f_{X}$. The idea is to use $G(\cdot)$ as a kind of "oblique" functional to force the density toward the shape of the beta $(4,4)$ density. This raises the issue of whether $f_{Y}\left(\cdot, \lambda_{0}\right)$ is easier to estimate than $f_{X}(\cdot)$. We assume that the left endpoint of $\Lambda$ gives the identical transformation, that is, $g_{\min (\Lambda)}(x) \equiv x$. This means that $\lambda=\min (\Lambda)$ corresponds to the untransformed density $f_{X}(\cdot)$, which is thus included in the family of transformed densities $\left\{f_{Y}\right\}_{\lambda \in \Lambda}$. We assume here that $\Lambda$ is compact so that an optimal parameter exists. Without loss of generality, we also assume that $\Lambda$ is one dimensional. One can then further assume that $\Lambda=\left[0, \lambda_{M}\right]$, where $\lambda_{M} \in(0,1)$ and that $g_{0}(x) \equiv x$. If this is not true, we just do a linear reparametrization of $\Lambda, \lambda \longrightarrow(\lambda-\min (\Lambda)) / 2 *(\max (\Lambda)-\min (\Lambda))$ to $\operatorname{make}$ it true. Now if $\lambda_{0}>0$, then $G\left(f_{Y}\left(\cdot, \lambda_{0}\right)\right)<G\left(f_{X}(\cdot)\right)$. That means transforming $f_{X}$ into $f_{Y}\left(\cdot, \lambda_{0}\right)$ by $g_{\lambda_{0}}(\cdot)$ provides an easier estimation setting, which allows improved performance. If, on the other hand, $\lambda_{0}=0$, then either no transformation, or transformation by a different family is needed.

We now define the following target function of $\lambda \in \Lambda$

$$
L(\lambda)=G\left(f_{Y}(\cdot, \lambda)\right)=\sigma\left(f_{Y}(\cdot, \lambda)\right)\left(R\left(f_{Y}^{\prime \prime}(\cdot, \lambda)\right)\right)^{1 / 5}
$$

and set $Y_{i}=g_{\lambda}\left(X_{i}\right), i=1,2, \ldots, n$. Then $Y_{1}, \ldots, Y_{n}$ are i.i.d. and each has the same density function $f_{Y}$ given in $(2.2)$. Since $L(\lambda)$ is minimized at $\lambda_{0}$, an approach to estimation of $\lambda_{0}$ is based on estimation of $L(\lambda)$. We estimate $\lambda_{0}$ with $\hat{\lambda}$, the minimizer of

$$
\widehat{L}(\lambda)=\sigma_{\widehat{Y}}(\lambda) \int\left[\hat{f}_{Y}^{\prime \prime}(y, b, \lambda)^{2}\right] d y
$$

where $\sigma_{\widehat{Y}}(\lambda)$ is the standard deviation of $Y_{1}, \ldots, Y_{n}$,

$$
\widehat{f}_{Y}^{\prime \prime}(y, b, \lambda)=\frac{d^{2}}{d y^{2}} \hat{f}_{Y}(y, b, \lambda)=1 / n \sum_{j=1}^{n} \varphi_{b}^{(2)}\left(y-Y_{j}\right)
$$


and $\varphi$ denotes the Gaussian kernel, i.e., $\varphi(u)=\frac{1}{\sqrt{2 \pi}} e^{-\frac{u^{2}}{2}}$ with $\varphi_{b}^{(k)}(u)=b^{-(k+1)} \varphi^{(k)}(u / b)$. Here $b$ is a pilot bandwidth used solely for $\hat{\lambda}$. Because $\Lambda$ is compact and $\hat{L}(\lambda)$ is continuous in $\lambda$ [under some mild assumptions, see Section 2.1 of Yang (1995a)], $\hat{\lambda}$ clearly exists. We do not need $\hat{\lambda}$ to be unique.

Once $\hat{\lambda}$ is obtained, one also gets $\hat{h}_{*}$, an estimate of $h_{*}$, the optimal bandwidth as in (2.5), except the underlying density is $f_{Y}\left(\cdot, \lambda_{0}\right)$. This $\hat{h}_{*}$ can be the Sheather-Jones plug-in bandwidth $h_{S J}$, the Park-Marron plug-in bandwidth $h_{P M}$ or other versions, as discussed in Jones, Marron and Sheather (1992). Then one gets the following estimate of $f_{X}(x)$

$$
\hat{f}_{X}\left(x, \hat{h}_{*}, \hat{\lambda}\right)=n^{-1} \sum_{j=1}^{n} g_{\hat{\lambda}}^{\prime}(x) \varphi_{\hat{h}_{*}}\left[g_{\hat{\lambda}}(x)-g_{\hat{\lambda}}\left(X_{j}\right)\right] .
$$

As in Wand, Marron and Ruppert (1991), the global performance of the estimator (2.10) is conveniently assessed by the AMISE of $\hat{f}_{Y}\left(\cdot, \hat{h}_{*}, \hat{\lambda}\right)$ which is an estimate of

$$
A M I S E_{Y}\left(h_{*}, \lambda_{0}\right)=\inf _{h>0} A M I S E_{Y}\left(h, \lambda_{0}\right)=C n^{-4 / 5} L\left(\lambda_{0}\right)^{1 / 5}=C n^{-4 / 5} \min _{\lambda \in \Lambda} L(\lambda)^{1 / 5}
$$

which is consistent with our view here because both amount to minimizing the same function $L(\lambda)$.

This approach leads to a big, computational advantage. The binning ideas of Scott and Terrell (1987), Fan and Marron (1994) are very straightforward to implement for $\hat{f}_{Y}\left(\cdot, \hat{h}_{*}, \hat{\lambda}\right)$. One can bin $Y_{1}, \ldots, Y_{n}$ and then estimate $\hat{f}_{Y}\left(\cdot, h_{*}, \lambda\right)$, which is simply a binned implementation of an ordinary kernel estimator. From $\widehat{f}_{Y}\left(\cdot, \widehat{h}_{*}, \hat{\lambda}\right)$ to $\widehat{f}_{X}\left(\cdot, \hat{h}_{*}, \hat{\lambda}\right)$ only takes the simple step of formula (2.10). The computation of $\hat{f}_{X}\left(\cdot, \hat{h}_{*}, \hat{\lambda}\right)$ is thus as interactive as the ordinary kernel estimation method, except for the steps needed to estimate $\lambda_{0}$ with $\hat{\lambda}$.

To complete the description of the implementation procedure, we now give 2 data-driven bandwidths $b$ that can be used in the pilot estimator $\widehat{L}(\lambda)$ :

1. The diagonals-out bandwidth of Park-Marron, which minimizes the asymptotic mean squared error of estimating $\int f_{Y}^{\prime \prime 2} d y$ by $\frac{1}{n^{2}} \sum_{i \neq j} \varphi_{\sqrt{2} b}^{(4)}\left(Y_{i}-Y_{j}\right)$

$$
b_{P M}=C_{1}\left(f_{Y}\right) D_{1}(\varphi) n^{-\frac{2}{13}}
$$

2. The diagonals-in bandwidth of Sheather-Jones, which minimizes the asymptotic mean squared error of estimating $\int f_{Y}^{\prime \prime 2} d y$ by $\frac{1}{n^{2}} \sum_{i, j=1}^{n} \varphi_{\sqrt{2} b}^{(4)}\left(Y_{i}-Y_{j}\right)$

$$
b_{S . J}=C_{2}\left(f_{Y}\right) D_{2}(\varphi) n^{-\frac{1}{7}}
$$


The $b_{S . J}$ was used in computing the Sheather-Jones bandwidth, as $b_{P M}$ is used in computing the Park-Marron bandwidth. An account of $b_{S J}, b_{P M}$, and the functionals $C_{1}(\cdot), C_{2}(\cdot), D_{1}(\cdot)$, and $D_{2}(\cdot)$ is in Jones, Marron and Sheather (1992). The pilot bandwidth $b_{P M}$ was used in Wand, Marron and Ruppert (1991). Since the Sheather-Jones bandwidth is preferred over the Park-Marron bandwidth in overall performance, we use $b_{S J}$ instead of $b_{P M}$ in our simulation studies and applications.

About the behavior of $\hat{\lambda}$ as an estimator of $\lambda_{0}$, we know that under certain regularity conditions on $f_{X}, g_{\lambda}(\cdot)$ and the pilot bandwidth $b, \hat{\lambda}$ converges consistently to $\lambda_{0}$. Also, $\hat{\lambda}$ converges to $\lambda_{0}$ in probability with asymptotic bias of order $b^{2}$ and asymptotic variance of order $1 /\left(n+n^{2} b^{9}\right)$. For our algorithm that uses $b_{S J}, \hat{\lambda}$ has bias of order $n^{-\frac{2}{7}}$ and variance of order $n^{-\frac{5}{7}}$. Proofs of these results are in Chapter 6 of Yang (1995a).

For the choice of transformation family, we define explicitly the Johnson Families

$$
\begin{gathered}
g_{1, \lambda}(x)=\frac{1}{c J} \ln (1+c J x), 0<\lambda \leq \lambda_{M}, \\
g_{2, \lambda}(x)=\frac{1}{c} \ln \left(c x+\sqrt{1+c^{2} x^{2}}\right), 0<\lambda \leq \lambda_{M}, \\
g_{3, \lambda}(x)=\frac{1}{2 c} \ln [(1+c x) /(1-c x)], 0<\lambda \leq \lambda_{M},
\end{gathered}
$$

where $J= \pm 1, c=\lambda^{p} /\left(1-\lambda^{p}\right), g_{i, 0}(x) \equiv x$, for $i=1,2,3$, and $p \geq \frac{1}{2}$ is a tuning constant which is needed for smoothness of $g_{\gamma, \lambda}(x)$ as a function of $\lambda$, as can be seen in Theorem 5.2 and its proof. Another way of writing these is:

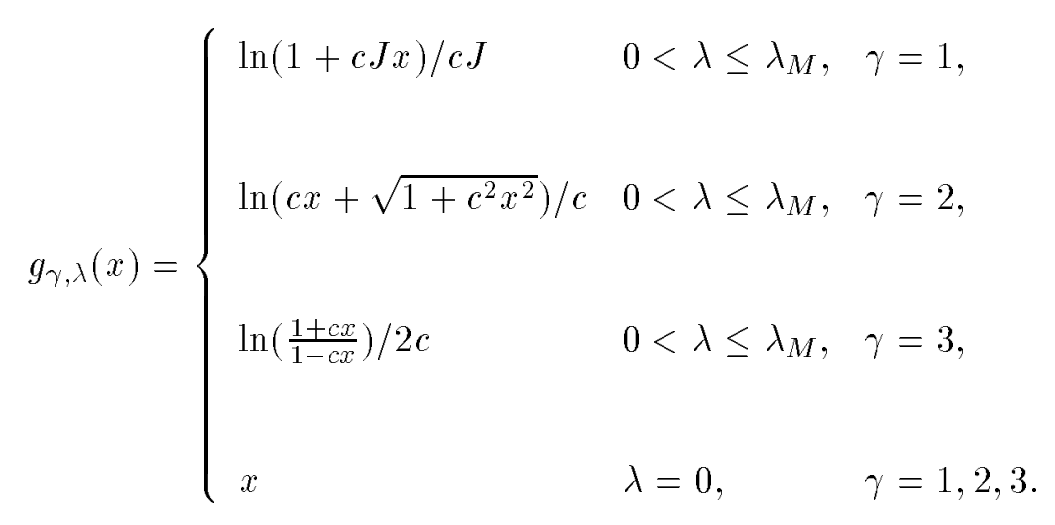

which is equivalent to pooling several families together by adding a discrete parameter ( $\gamma$ here). We use $c=\lambda^{p} /\left(1-\lambda^{p}\right)$ instead of $\eta$ so that $\eta$ lies entirely in $[0,1)$ yet $c$ lies in the full interval $[0, \infty)$. This is important since larger $c$ means more drastic transformation. For Johnson Family, see Johnson (1949).

To provide some intuition as to why we use these 3 families, we include in Figure 3 four pictures, each overlays six density curves that are the results of applying the inverse of one of the Johnson 
Families to the standard normal density, using equally spaced $\eta$ values. The curve $\lambda=0$ is the standard normal density.

As $\eta$ increases, the curve becomes more and more skewed under Johnson Family 1. This suggests that when a density $f_{X}$ has high skewness, an optimal transformation from the Johnson Family 1 will transform it into a near normal shape. Note that $J=-1$ and 1 handles skewness of both orientations. Similarly, Johnson Family 2 deals with density shapes that are kurtotic, i.e., having large probability mass near the mean and in the tails. Johnson Family 3 deals with density shapes that have small kurtosis, i.e., those having less probability mass in the center and in the tails. Later in Section 5, we show that the Johnson Families are indeed able to improve the estimation of most density functions.

Since there are three Johnson Families, we describe the TKDE method when several families are used at the same time. Suppose that we have $m$ families of transformations $\left\{g_{j}\right\}, j=1,2,3, \ldots, m$, each with its own range of parameters $\Lambda_{j}=\left[0, \lambda_{j, M}\right], g_{j}(x, 0) \equiv x$, for $j=1,2,3, \ldots, m$. Suppose also that $\lambda_{j}=\arg \min _{\lambda \in \Lambda_{j}} L_{j}(\lambda)$, where $L_{j}(\lambda)$ is defined as in $(2.8)$ and $L_{j}=L_{j}\left(\lambda_{j}\right), j=1,2,3, \ldots, m$. Let

$\hat{\lambda}_{j}=\arg \min _{\lambda \in \Lambda_{j}} \widehat{L}_{j}(\lambda), j=1,2,3, \ldots, m$, where $\widehat{L}_{j}(\lambda)$ is defined as in $(2.9)$. Let $\hat{l}_{j}=\widehat{L}_{j}\left(\hat{\lambda}_{j}\right)$ be the pilot estimator of $L, j=1,2, \ldots, m$. To decide which family is more suitable for transforming density $f_{X}$, we use the following rules.

\section{Family Selection Rules:}

- Theoretical rule: use family $j$ if $L_{j}=\min \left\{L_{k}, 1 \leq k \leq m\right\}$,

- Data-driven rule: with a given sample, use family $j$ if $\hat{l}_{j}=\min \left\{\hat{l}_{k}, 1 \leq k \leq m\right\}$.

So theoretically, one chooses family $j$ over family $k$ if $L_{j}<L_{k}$. Here the convention is that when there are ties among the $L_{k}$ 's, one can order arbitrarily the tied families. The data-driven rule has the same convention about ties.

\section{Theorem 2.1}

$$
P\left[\left(\hat{l}_{j}-\hat{l}_{k}\right)\left(L_{j}-L_{k}\right)<0 \text { for some } 1 \leq j, k \leq m, j \neq k\right] \rightarrow 0 \text {, as } n \rightarrow \infty .
$$

Therefore, the probability of not selecting the family with lowest $L$ value is asymptotically zero.

This theorem guarantees that the Data-driven rule is asymptotically the same as the Theoretical rule. The proof is based on the fact that $\hat{l}_{j} \rightarrow L_{j}$ in probability, for $j=1,2, \ldots, m$, as $n \rightarrow \infty$. For a proof, see Section 7.2 of Yang (1995a).

The method we just finished describing in this section will be used throughout the rest of the paper. 


\section{APPLICATIONS}

In this section, we give two examples of real data, see Chapter 5 of Yang (1995a) for more. We first analyze the Adoption Visa data in Section 1. Recall that $L(\lambda)=G\left(f_{Y}(\cdot, \lambda)\right)$ as defined in $(2.8)$.

Figure 4 shows that $G(\cdot)$ is reduced from about 1.81 to about 0.743 . Most of the reduction happens in Step 1. Both steps were by Johnson Family 1 because the data is very skewed. Figure 5 shows that the "Transformed Twice" estimate in the $Z$-space, has little curvature because $G\left(f_{Z}\right)$ has nearly achieved the minimum of 0.68 as shown in the right side of Figure 5 . There is little to be gained to further transform $Z$.

The second dataset consists of the shooting percentages (percentages of shots taken that score) of 105 National Hockey League players [National Hockey League (1992)].

Figure 6 shows that the estimate without transformation missed the first peaks (or created spurious peaks in the right tail, if a smaller bandwidth was used), while the estimates with transformation (once or twice) were able to get exactly the three peaks and remain smooth in the tail. We were informed by personal communication from J. S. Simonoff that the three peaks correspond to three groups of players at different levels of proficiency.

\section{SIMULATION STUDY}

To understand the effectiveness and the asymptotics of the transformation method, we present in this section results of a simulation study on one normal mixture density, the Skewed M-shape density shown in Figure 7. It is of the form

$$
f_{X}(x)=\sum_{j=1}^{k} w_{j} \varphi_{\sigma_{j}}\left(x-\mu_{j}\right)=\sum_{j=1}^{k} w_{j} \frac{1}{\sqrt{2 \pi} \sigma_{j}} \epsilon^{-\frac{\left(x-\mu_{j}\right)^{2}}{2 \sigma_{j}^{2}}},
$$

where

$$
\begin{gathered}
w^{T}=\left(w_{1}, w_{2}, \ldots, w_{k}\right)=\frac{1}{16}(1,1, \ldots, 1,8)_{1 \times 9}, \sigma^{T}=\left(\sigma_{1}, \sigma_{2}, \ldots, \sigma_{k}\right)=\left(1, \frac{2}{3}, \ldots,\left(\frac{2}{3}\right)^{7}, 1\right)_{1 \times 9}, \\
\mu^{T}=\left(\mu_{1}, \mu_{2}, \ldots, \mu_{k}\right)=\left(3 \times 1-1,3 \times \frac{2}{3}-1, \ldots, 3 \times\left(\frac{2}{3}\right)^{7}-1,0\right)_{1 \times 9} .
\end{gathered}
$$

The simulation results on four other normal mixtures: the M-shape, the Strongly Skewed, the Standard Normal, and the Kurtotic are not included here. The lessons of those are parallel to that of the Skewed M-shape [Chapter 4 of Yang (1995a)]. The normal mixtures comprise a very broad class. The Skewed M-shape and the M-shape are new additions to the fifteen normal mixtures of Marron 
and Wand (1992), which included the other three mentioned above. Figure 7 shows the Skewed Mshape density ("True") together with estimates by three methods: the "Untransformed" refers to the global Sheather-Jones bandwidth estimate; the "Transformed" refers to the estimate by applying transformation from $X$ to $Y$ with Johnson Family 1; the "Transformed Twice" refers to the estimate by applying transformation in two consecutive steps: from $X$ to $Y$ and then from $Y$ to $Z$ by Johnson Family 3.

In Figure 7, the values "SSEU", "SSE1", "SSE2" are the sum of the squared errors of the "Untransformed", the "Transformed", and the "Transformed Twice" estimates, with respect to the "True" density. The number "NOBS" is the number of observations. Here we have applied the binned implementation with the number of bins $=401$ [Fan and Marron (1994)]. The "+"'s represent 26 points on the $X$ scale that become equally spaced in the $Y$ scale after transformation. We see that $S S E U>$ $S S E 1>S S E 2$, which shows that the "Transformed" estimator is better than the "Untransformed" while the "Transformed Twice" is the best of all three.

The theoretical $G(\cdot)$-values of transformed densities under all three Johnson Families are plotted in Figure 8 (the parameters for all three families are adjusted to the same scale). It shows why the Johnson Family 1 is the family used to transform the Skewed M-shape Density because it reduces the $G(\cdot)$-value the most: from about 5.6 to 1.4. This is because the Skewed M-shape Density is more skewed than kurtotic compared to the beta $(4,4)$.

Now we come back to the pseudo dataset used in Figure 7 . We give here two pictures to show what happens in the transformed scales. Figure 7 and Figure 9 show how transformation by Family 1 and then Family 3 were applied to the pseudo dataset successively. The skewness was corrected first and then the kurtosis. Our algorithm was able to detect that skewness is the more dominant feature than kurtosis and needs to be dealt with first. Finally, Figure 10 shows how the reduction of $G(\cdot)$ in the two transformation steps.

To better understand the variability of our estimates across datasets, we generated 500 pseudo datasets from the Skewed M-Shape density, with sample sizes equal to 100, 1000, 10000, respectively, and applied transformation method to these 1500 datasets. The curves in Figure 11 are kernel estimates of the densities of the $G(\cdot)$-values (bottom row) and the log integrated squared errors (" $\log (I S E)$ )"'s (top row). The values of " $M L X "$ " " $M L Y$ " and " $M L Z$ " represent the means of the $500 G(\cdot)$-values that correspond to the three methods: "Untransformed", "Transformed", and "Transformed Twice".

We observe that the "Transformed" curve is always to the left of the "Untransformed" and to the right of the "Transformed Twice", thus the $G(\cdot)$-values become less after applying transformation. 
Both graphically and in terms of " $M L X$ ", " $M L Y$ " and " $M L Z$ ", the difference between the "Untransformed" and the "Transformed" is greater than that between the "Transformed" and "Transformed Twice". Thus most of the reduction of $G(\cdot)$ happens in transformation step one. The conclusion for the $\log (I S E)$ 's is similar: a huge improvement from "Untransformed" to "Transformed" and a smaller yet non-negligible improvement from "Transformed" to "Transformed Twice".

We use Table 1 to show how often the right transformation family is selected. The Johnson Family 1 was selected in Step 1 with relative frequencies of $107 / 500=0.214,440 / 500=0.88$, and $500 / 500=1$. In Step 2, the relative frequencies of selecting the Johnson Family 3 are: $347 / 500=0.694$, $440 / 500=0.88$, and $500 / 500=1$. This table shows that the probability of selecting the right family tends to 1 rather rapidly.

\begin{tabular}{rrrrrrrr}
\multicolumn{3}{c}{ In Step 1 } & \multicolumn{5}{c}{ In Step 2 } \\
NOBS $=$ & 100 & 1000 & 10000 & NOBS $=$ & 100 & 1000 & 10000 \\
FAMILY 1 & 107 & 440 & 500 & FAMILY 1 & 149 & 60 & 0 \\
FAMILY 2 & 15 & 0 & 0 & FAMILY 2 & 4 & 0 & 0 \\
FAMILY 3 & 378 & 60 & 0 & FAMILY 3 & 347 & 440 & 500
\end{tabular}

Table 1: The Skewed M-shape Density, the frequencies

Figure 12 displays kernel density estimates of those $\widehat{\lambda}$-values when the right transformation families are used. It shows that $\hat{\lambda}$ converges rapidly as the sample size goes from 100 to 1000 to 10000 .

\section{THE EFFECTIVENESS OF JOHNSON FAMILIES}

In this section, we give results that for any density $f$ satisfying certain rather mild conditions, $G(f)$ can be reduced by applying at least one of the three Johnson Families.

To conform to the notation of Johnson Families in Section 2, we use $\eta$ instead of $\lambda$ for the parameter. Recall from Section 2 that

$$
G\left(f_{X}\right)=\sigma\left(f_{X}\right)\left(R\left(f_{X}^{\prime \prime}\right)\right)^{1 / 5}=\sigma_{f_{X}}\left(R\left(f_{X}^{\prime \prime}\right)\right)^{1 / 5}
$$

is the functional to be minimized through transformation. The univariate function to be minimized is $L(\lambda)=\int f_{Y}^{\prime \prime}(y)^{2} d y=G\left(f_{Y}\right)^{5}$, where $Y=g_{\lambda}(X)$. Denote the minimizer by $\eta_{0}$. Now the parameters are in $\Pi=\left[0, \lambda_{M}\right]$, where $\eta_{M} \in(0,1), g_{0}(x) \equiv x$, and $L(0)=G\left(f_{X}\right)^{5}$. A sufficient condition for $\left\{g_{\lambda}\right\}$ 
to reduce $G(f)$ is that $L^{\prime}(0)<0$, which implies that there is a small enough $\epsilon$ such that $L(\epsilon)<L(0)$, thus $L\left(\lambda_{0}\right) \leq L(\epsilon)<L(0)$. To simplify notation, we use in this section

$$
L(\lambda)=\sigma_{Y}^{10} R\left(f_{Y}^{\prime \prime}\right)^{2}=G\left(f_{Y}\right)^{10}=\left[\int g_{\lambda}(x)^{2} f_{X}(x) d x-\left(\int g_{\lambda}(x) f_{X}(x) d x\right)^{2}\right]^{5}\left(\int f_{Y}^{\prime \prime}(y)^{2} d y\right)^{2},
$$

which is equivalent to the previous definition of $L$. We assume the following:

- A1. that $g_{\lambda}$ is jointly $C^{3}$ for $x \in S\left(f_{X}\right)$ and $\lambda \in \Lambda$;

- A2. that $f_{Y} \in S^{3}(R)$, the space of third order rapidly decreasing functions, for every $\lambda \in \Lambda$. This means that $f_{Y}$ is $C^{3}$ and that for any $k=1,2,3, \ldots$ and $l=0,1,2,3, \lim _{|y| \rightarrow \infty}|y|^{k} f_{Y}^{(l)}(y)=0$;

- A3. that $\mu_{X}=\int x f_{X}(x) d x=0$.

Assumption A1 is satisfied by the Johnson Families. Assumption A2 is satisfied by densities such as normal mixture and exponential. Assumption A3 is just for convenience, if it is not satisfied, a translation of $f_{X}$ can achieve it.

Theorem 5.1 Under assumptions A1, A2, and A3,

$$
\begin{aligned}
& L^{\prime}(0)=2 \sigma_{X}^{8} R\left(f_{X}^{\prime \prime}\right)\left\{\left.5 R\left(f^{\prime \prime}\right) \int x \frac{d}{d \eta} g_{\lambda}(x)\right|_{\lambda=0} f_{X}(x) d x+2 \sigma_{X}^{2} \int f_{X}^{\prime \prime}(x)\left[f_{X}^{(3)}(x) x\right.\right. \\
& \left.\left.+3 f_{X}^{(2)}(x) \frac{d}{d \eta} g_{\lambda}^{-1}(x)+3 f_{X}^{\prime}(x) \frac{d}{d \eta}\left(g_{\lambda}^{-1}\right)^{\prime \prime}(x)+f_{X}(x) \frac{d}{d \eta}\left(g_{\lambda}^{-1}\right)^{\prime \prime \prime}(x)\right]\left.\right|_{\lambda=0} d x\right\}
\end{aligned}
$$

that is,

$$
\begin{aligned}
L^{\prime}(0)= & 2 \sigma_{X}^{8} R\left(f_{X}^{\prime \prime}\right)\left\{\left.5 \int x \frac{d}{d \eta} g(x)\right|_{\lambda=0} f_{X}(x) d x \int f^{\prime \prime}(x)^{2} d x+2 \int x^{2} f_{X}(x) d x \int f_{X}^{\prime \prime}(x)\left[f_{X}^{(3)}(x) x\right.\right. \\
& \left.\left.+3 f_{X}^{(2)}(x) \frac{d}{d \eta} g_{\lambda}^{-1}(x)+3 f_{X}^{\prime}(x) \frac{d}{d \eta}\left(g_{\lambda}^{-1}\right)^{\prime \prime}(x)+f_{X}(x) \frac{d}{d \eta}\left(g_{\lambda}^{-1}\right)^{\prime \prime \prime}(x)\right]\left.\right|_{\lambda=0} d x\right\} .
\end{aligned}
$$

Theorem 5.2 Under assumptions A1, A2, and A3, define

$$
\begin{gathered}
A\left(f_{X}\right)=-\frac{5}{3} \sigma_{X}^{8} R\left(f_{X}^{\prime \prime}\right)\left\{\int x^{4} f_{X} R\left(f_{X}^{\prime \prime}\right)-3 \sigma_{X}^{2}\left[\int x^{2}\left(f_{X}^{\prime \prime}\right)^{2}-2 \int\left(f_{X}^{\prime}\right)^{2}\right]\right\} \\
=-\frac{5}{3} \sigma_{X}^{8} R\left(f_{X}^{\prime \prime}\right)\left\{\int x^{4} f_{X}(x) d x \int\left(f_{X}^{\prime \prime}(x)\right)^{2} d x-3 \int x^{2} f_{X}(x) d x\left[\int x^{2}\left(f_{X}^{\prime \prime}(x)\right)^{2} d x-2 \int\left(f_{X}^{\prime}(x)\right)^{2} d x\right]\right\}
\end{gathered}
$$

and

$$
\begin{gathered}
B\left(f_{X}, J\right)=-J \frac{5}{2} \sigma_{X}^{8} R\left(f_{X}^{\prime \prime}\right)\left\{\int x^{3} f_{X} R\left(f_{X}^{\prime \prime}\right)-2 \sigma_{X}^{2} \int x\left(f_{X}^{\prime \prime}\right)^{2}\right\} \\
=-J \frac{5}{2} \sigma_{X}^{8} R\left(f_{X}^{\prime \prime}\right)\left\{\int x^{3} f_{X}(x) d x \int\left(f_{X}^{\prime \prime}(x)\right)^{2} d x-2 \int x^{2} f_{X}(x) d x \int x\left(f_{X}^{\prime \prime}(x)\right)^{2} d x\right\}
\end{gathered}
$$


(1) Let $p=1$, if $J=1$ and $S\left(f_{X}\right)$ is bounded from below, or if $J=-1$ and $S\left(f_{X}\right)$ is bounded from above, then for family $\left\{g_{1}\right\}$,

$$
L^{\prime}(0)=B\left(f_{X}, J\right)=-B\left(f_{X},-J\right)
$$

(2) Let $p=\frac{1}{2}$, for family $\left\{g_{2}\right\}$,

$$
L^{\prime}(0)=A\left(f_{X}\right)
$$

(3) Let $p=\frac{1}{2}$, if $S\left(f_{X}\right)$ is bounded, then for family $\left\{g_{3}\right\}$,

$$
L^{\prime}(0)=-2 A\left(f_{X}\right)
$$

Thus if $S\left(f_{X}\right)$ is bounded and $A\left(f_{X}\right)^{2}+B\left(f_{X}, 1\right)^{2}>0$, then at least one family out of $\left\{g_{1}\right\}$ (with both options of $J$ available $),\left\{g_{2}\right\}$ and $\left\{g_{3}\right\}$ can reduce the value of $\sigma_{X}^{10} R\left(f_{X}^{\prime \prime}\right)^{2}$.

Note that $A\left(f_{X}\right)^{2}+B\left(f_{X}, 1\right)^{2}=0$ happens very rarely because it requires both $A\left(f_{X}\right)$ and $B\left(f_{X}, 1\right)$ to be 0 . Theorem 5.2 guarantees that every compactly supported and third order smooth density $f_{X}$ can be made easier to estimate by one of the Johnson Families. As for infinitely supported densities, their truncation can be used. We have the following generalized version of Theorem 5.2,

Theorem 5.3 Under assumptions $A 1, A 2$, and $A 3$, and $A\left(f_{X}\right)^{2}+B\left(f_{X}, 1\right)^{2}>0$, there exist constants depending on $f_{X}, R_{0}>0, \epsilon>0$, and a certain Johnson Family $\left\{g_{\gamma, \lambda}\right\}$ such that for every $R>R_{0}$, if we denote by $\left(f_{X}\right)_{R}$ the truncation of $f_{X}$ to the interval $[-R, R]$, and by $\left(f_{Y}\right)_{R}$ the optimally transformed density from $\left(f_{X}\right)_{R}$ by $\left\{g_{\gamma, \lambda}\right\}$, then the following is true,

$$
\left|G\left(f_{X}\right)-G\left(\left(f_{X}\right)_{R}\right)\right|<\frac{\epsilon}{2} \text { and } G\left(\left(f_{Y}\right)_{R}\right)<G\left(\left(f_{X}\right)_{R}\right)-\epsilon
$$

therefore

$$
G\left(\left(f_{Y}\right)_{R}\right)<G\left(f_{X}\right)-\frac{\epsilon}{2}
$$

This result provides a theoretical basis for applying all three Johnson Families to infinitely supported densities.

As an example, consider densities beta $(k, k), k \geq 5$. It is easily shown that their $B(\cdot)$ 's are 0 . A direct calculation shows that their $A(\cdot)$ 's are all $<0$, therefore Johnson Family 3 is able to improve their kernel estimation. This is consistent with the fact that Family 3 reduces the kurtosis as shown in Figure 3 : As a density with high $k$ is transformed to a shape close to the optimal $k=4$, its kurtosis $\left(\frac{2 k+1}{2 k+3}\right)$ decreases as $k$ decreases. A special case $(k=\infty)$, i.e., the standard normal, has been a target of our simulation work. 


\section{CONCLUSIONS}

In view of the findings on the simulated and real examples in this paper and Chapters 4 and 5 of Yang (1995a), we conclude that:

1. One of the Johnson Families is suitable for transforming any given density as indicated by Theorem 5.2; as the sample size increases, our algorithm is able to select the right Johnson Family in most cases, as indicated by Theorem 2.1;

2. the estimates of the optimal parameter $\eta_{0}$ converge with satisfactory speed;

3. the $G(\cdot)$-values and the $I S E$ 's are reduced by each step of transformation, more in the first step than the second, and in most cases little can be gained to transform more;

4. the transformation is more effective on densities with sharp features; the effects are marginal otherwise yet there is some improvement.

Based on these conclusions, we recommend the iterated transformation method for kernel density estimation in most situations.

We also want to point out that although in this paper we have used exclusively the Johnson Families, any number of other families can be used simultaneously as well. In particular, it may be possible to combine the power transformation family used in Wand, Marron and Ruppert (1991), the kurtosis reducing family used in Ruppert and Wand (1992), and a third family that increases kurtosis. In such a setting, Theorem 2.1 guarantees that the best family will be automatically selected for any density. Further investigation will be needed, however, to establish the analog of Theorem 5.2 in order to show that such a union of families can effectively transform a wide range of density functions.

\section{APPENDIX}

We give here the proofs of Theorem 5.1 and Theorem 5.2. Proof of Theorem 5.3 is straightforward from Theorem 5.2, it is not included here.

Proof of Theorem 5.1. This is rather straightforward. Note for $\eta>0$ and $Y=g_{\lambda}(X)$,

$$
f_{Y}(y)=f_{X}\left(g_{\lambda}^{-1}(y)\right)\left(g_{\lambda}^{-1}\right)^{\prime}(y)
$$

and thus

$$
\frac{d}{d \eta} f_{Y}^{\prime \prime}(y)=f_{X}^{\prime \prime \prime}\left(g_{\lambda}^{-1}(y)\right) \frac{d}{d \eta} g_{\lambda}^{-1}(y)\left(\left(g_{\lambda}^{-1}\right)^{\prime}(y)\right)^{3}+3 f_{X}^{\prime \prime}\left(g_{\lambda}^{-1}(y)\right)\left(\left(g_{\lambda}^{-1}\right)^{\prime}(y)\right)^{2} \frac{d}{d \eta}\left(g_{\lambda}^{-1}\right)^{\prime}(y)
$$




$$
\begin{gathered}
+3 f_{X}^{\prime \prime}\left(g_{\lambda}^{-1}(y)\right)\left(g_{\lambda}^{-1}\right)^{\prime \prime}(y)\left(g_{\lambda}^{-1}\right)^{\prime}(y) \frac{d}{d \eta}\left(g_{\lambda}^{-1}\right)(y)+3 f_{X}^{\prime}\left(g_{\lambda}^{-1}(y)\right)\left(g_{\lambda}^{-1}\right)^{\prime \prime}(y) \frac{d}{d \eta}\left(g_{\lambda}^{-1}\right)^{\prime}(y) \\
+3 f_{X}^{\prime}\left(g_{\lambda}^{-1}(y)\right)\left(g_{\lambda}^{-1}\right)^{\prime}(y) \frac{d}{d \eta}\left(g_{\lambda}^{-1}\right)^{\prime \prime}(y)+f_{X}^{\prime}\left(g_{\lambda}^{-1}(y)\right)\left(g_{\lambda}^{-1}\right)^{\prime \prime \prime}(y) \frac{d}{d \eta}\left(g_{\lambda}^{-1}\right)(y)+f_{X}\left(g_{\lambda}^{-1}(y)\right) \frac{d}{d \eta}\left(g_{\lambda}^{-1}\right)^{\prime \prime \prime}(y) .
\end{gathered}
$$

When $\eta=0$, we have $g_{\lambda}^{-1}(y) \equiv y,\left(g_{\lambda}^{-1}\right)^{\prime}(y) \equiv 1,\left(g_{\lambda}^{-1}\right)^{(k)}(y) \equiv 0$ for $k>1$, therefore

$$
\left.f_{Y}^{\prime \prime}(y)\right|_{\lambda=0}=f_{X}^{\prime \prime}(y)(1)^{3}+3 f_{X}^{\prime}(y) \times 1 \times 0+f_{X}(y) \times 0=f_{X}^{\prime \prime}(y)
$$

and similarly

$$
\left.\frac{d}{d \eta} f_{Y}^{\prime \prime}(y)\right|_{\lambda=0}=f_{X}^{\prime \prime \prime}(y) \frac{d}{d \eta} g_{\lambda}^{-1}(y)+3 f_{X}^{\prime \prime}(y) \frac{d}{d \eta}\left(g_{\lambda}^{-1}\right)^{\prime}(y)+3 f_{X}^{\prime}(y) \frac{d}{d \eta}\left(g_{\lambda}^{-1}\right)^{\prime \prime}(y)+f_{X}(y) \frac{d}{d \eta}\left(g_{\lambda}^{-1}\right)^{\prime \prime \prime}(y) .
$$

We therefore conclude from (6.1) and (6.2) that

$$
\begin{aligned}
\left.\frac{d}{d \eta}\right|_{\lambda=0} & R\left(f_{Y}^{\prime \prime}\right)=\int 2 f_{X}^{\prime \prime}(x)\left(f_{X}^{\prime \prime \prime}(x) \frac{d}{d \eta} g_{\lambda}^{-1}(x)+3 f_{X}^{\prime \prime}(x) \frac{d}{d \eta}\left(g_{\lambda}^{-1}\right)^{\prime}(x)\right. \\
+ & \left.+3 f_{X}^{\prime}(x) \frac{d}{d \eta}\left(g_{\lambda}^{-1}\right)^{\prime \prime}(x)+f_{X}(x) \frac{d}{d \eta}\left(g_{\lambda}^{-1}\right)^{\prime \prime \prime}(x)\right)\left.\right|_{\lambda=0} d x
\end{aligned}
$$

Now note also that

$$
\left.\frac{d}{d \eta}\right|_{\lambda=0}\left(\sigma_{Y}^{2}\right)=\left.\frac{d}{d \eta}\right|_{\lambda=0}\left(\int y^{2} f_{Y}(y) d y-\left(\int y f_{X}(y) d y\right)^{2}\right)=\left.\int 2 x \frac{d}{d \eta} g_{\lambda}(x)\right|_{\lambda=0} f_{X}(x) d x,
$$

because by assumption $\mathrm{A} 3, \mu_{X}=0$. Now $L(\lambda)=\sigma_{Y}^{10} R\left(f_{Y}^{\prime \prime}\right)^{2}$, so

$$
\left.\frac{d}{d \eta}\right|_{\lambda=0} L(\lambda)=\left.5 \sigma_{Y}^{8} \frac{d}{d \eta}\right|_{\lambda=0}\left(\sigma_{Y}^{2}\right) R\left(f_{Y}^{\prime \prime}\right)^{2}+\left.2 \sigma_{Y}^{10} \frac{d}{d \eta}\right|_{\lambda=0} R\left(f_{Y}^{\prime \prime}\right) R\left(f_{Y}^{\prime \prime}\right) .
$$

Plugging in (6.3) and (6.4) directly completes the proof. Q. E. D.

To prove Theorem 5.2, we need some preliminary results. As a convention, set $\eta_{p}=\frac{p}{\lambda\left(1-\lambda^{p}\right)}$ in what follows. The next lemma is proved in Yang (1995a) [Lemma 7.1.2 on page 160].

Lemma 6.1 For Johnson Family 1, $g_{1, \lambda}(x)=\frac{1}{c J} \ln (1+c J x)$

$$
\frac{d}{d \eta} g_{1, \lambda}(x)=\left\{\begin{array}{ll}
\lambda_{p}\left(x g_{1, \lambda}^{\prime}(x)-g_{1, \lambda}(x)\right), \\
-\frac{J}{2} x^{2},
\end{array} \quad \frac{d}{d \eta} g_{1, \lambda}^{-1}(x)= \begin{cases}\lambda_{p}\left(x\left(g_{1, \lambda}^{-1}\right)^{\prime}(x)-g_{1, \lambda}^{-1}(x)\right) & \lambda>0, \\
0, & \lambda=0, p=1, \\
\frac{J}{2} x^{2} & \lambda=0, p>1\end{cases}\right.
$$

$$
\frac{d}{d \eta}\left(g_{1, \lambda}^{-1}\right)^{\prime}(x)=\left\{\begin{array}{ll}
\lambda_{p} x\left(g_{1, \lambda}^{-1}\right)^{\prime \prime}(x), \\
J x,
\end{array} \quad \frac{d}{d \eta}\left(g_{1, \lambda}^{-1}\right)^{\prime \prime}(x)= \begin{cases}\lambda_{p}\left(x\left(g_{1, \lambda}^{-1}\right)^{\prime \prime \prime}(x)+\left(g_{1, \lambda}^{-1}\right)^{\prime \prime}(x)\right) & \lambda>0, \\
J & \lambda=0, p=1, \\
0 & \lambda=0, p>1\end{cases}\right.
$$




$$
\frac{d}{d \eta}\left(g_{1, \lambda}^{-1}\right)^{\prime \prime \prime}(x)= \begin{cases}\lambda_{p}\left(x\left(g_{1, \lambda}^{-1}\right)^{\prime \prime \prime \prime}(x)+2\left(g_{1, \lambda}^{-1}\right)^{\prime \prime \prime}(x)\right) & \lambda>0, \\ 0 & \lambda=0, p=1, \\ 0 & \lambda=0, p>1 .\end{cases}
$$

Proof of Theorem 5.2. We prove only (1) of the Theorem, the other parts are similar. Substitute for the $\frac{d}{d \eta}$ terms in the formula of Theorem 5.1 their respective expressions as found in Lemma 6.1, we have

$$
\begin{gathered}
L^{\prime}(0)=J 2 \sigma_{X}^{8} R\left(f_{X}^{\prime \prime}\right)\left[-\frac{5}{2} \int x^{3} f_{X}(x) d x \int f^{\prime \prime}(x)^{2} d x\right. \\
\left.+2 \int x^{2} f_{X}(x) d x \int\left(f_{X}^{\prime \prime}(x) f_{X}^{\prime \prime \prime}(x) \frac{1}{2} x^{2}+3 f_{X}^{\prime \prime}(x)^{2} x+3 f_{X}^{\prime}(x) f_{X}^{\prime \prime}(x)\right) d x\right],
\end{gathered}
$$

note that $\int 3 f_{X}^{\prime}(x) f_{X}^{\prime \prime}(x) d x=\left.\frac{3}{2} f_{X}^{\prime}(x)^{2}\right|_{-\infty} ^{+\infty}=0$ by the assumption A2 which makes $\lim _{x \rightarrow \pm \infty} f_{X}^{\prime}(x)^{2}=0$, and by the same token, integrating by parts gives

$$
\int\left(f_{X}^{\prime \prime}(x) f_{X}^{\prime \prime \prime}(x) \frac{1}{2} x^{2} d x=\left.\frac{1}{2} f_{X}^{\prime \prime}(x)^{2} \frac{1}{2} x^{2}\right|_{-\infty} ^{+\infty}+\int \frac{1}{2}\left(-f_{X}^{\prime \prime}(x)^{2}\right) x d x=\int \frac{1}{2}\left(-f_{X}^{\prime \prime}(x)^{2}\right) x d x .\right.
$$

Now a little reduction gives the result,

$$
L^{\prime}(0)=-J \frac{5}{2} \sigma_{X}^{8} R\left(f_{X}^{\prime \prime}\right)\left\{\int x^{3} f_{X} R\left(f_{X}^{\prime \prime}\right)-2 \sigma_{X}^{2} \int x\left(f_{X}^{\prime \prime}\right)^{2}\right\}=B\left(f_{X}, J\right),
$$

which proved (1) of the Theorem.Q. E. D.

\section{References}

1. Chatterjee, S., Handcock, M.S., and Simonoff, J. S. (1995), A Casebook for a First Course in Statistics, John Wiley and Sons, New York.

2. Devroye, L. and Györfi, L. (1985), Nonparametric Density Estimation : The $L_{1}$ View, New York: John Wiley.

3. Fan, J., Marron, J. S. (1994), Fast Implementations of Nonparametric Curve Estimators, Journal of Computational and Graphical Statistics, 3(1), 35-56.

4. Johnson, N. L. (1949), Systems of Frequency Curves Generated by Methods of Translation, Biometrika, Volume XXXVI, 149-176.

5. Jones, M. C., Marron, J. S. and Sheather, S. J. (1992), Progress in Data-based Bandwidth Selection for Kernel Density Estimation, Mimeo Series \#2088, Institute of Statistics, University of North Carolina. 
6. Marron, J. S. and Wand, M. P. (1992), Exact Mean Integrated Squared Error, The Annals of Statistics, 20, 712-736.

7. National Hockey League (1992), The National Hockey League Official Guide and Record Book 1992-93, Triumph Books, Chicago.

8. Park, B. U., Chung, S. S. and Seog, K. H. (1992), An Empirical Investigation of the Shifted Power Transformation Method in Density Estimation, Computational Statistics \& Data Analysis, North Holland, 14, 183-191.

9. Ruppert, D. and Cline, D. B. H. (1994), Bias Reduction in Kernel Density Estimation, The Annals of Statistics, 22, 185-210.

10. Ruppert, D. and Wand, M. P. (1992), Correcting for Kurtosis in Density Estimation, Australian Journal of Statistics, 34, 19-29.

11. Scott, D. W. (1992), Multivariate Density Estimation, John Wiley \& Sons, Inc.

12. Scott, D. W. and Terrell, G. R. (1987), Biased and Unbiased Cross-Validation in Density Estimation, Journal of the American Statistical Association, 82, 1131-1146.

13. Silverman, B. W. (1986), Density Estimation for Statistics and Data Analysis, New York: Chapman and Hall.

14. Terrell, G. R. (1990), The Maximal Smoothing Principle in Density Estimation, Journal of the American Statistical Association, 85, 470-477.

15. Wand, M. P. and Jones, M. C. (1995), Kernel Smoothing, London: Chapman and Hall.

16. Wand, M. P. and Devroye, L. (1993), How Easy Is a Given Density to Estimate?, Computational Statistics \& Data Analysis, 16, 311-323.

17. Wand, M. P., Marron, J. S. and Ruppert, D. (1991). Transformations in Density Estimation, Journal of the American Statistical Association, 86, 343-361.

18. Yang, L. (1995a), Transformation-Density Estimation, Doctoral Dissertation, Department of Statistics, University of North Carolina.

19. Yang, L. (1995b), Root-n Convergent Transformation-Kernel Density Estimation, available at http://wotan.wiwi.hu-berlin.de/pub/papers/sfb373/dpsfb960094.ps.Z. 
Figure 1: Untransformed Estimates, the Adoption Visa data

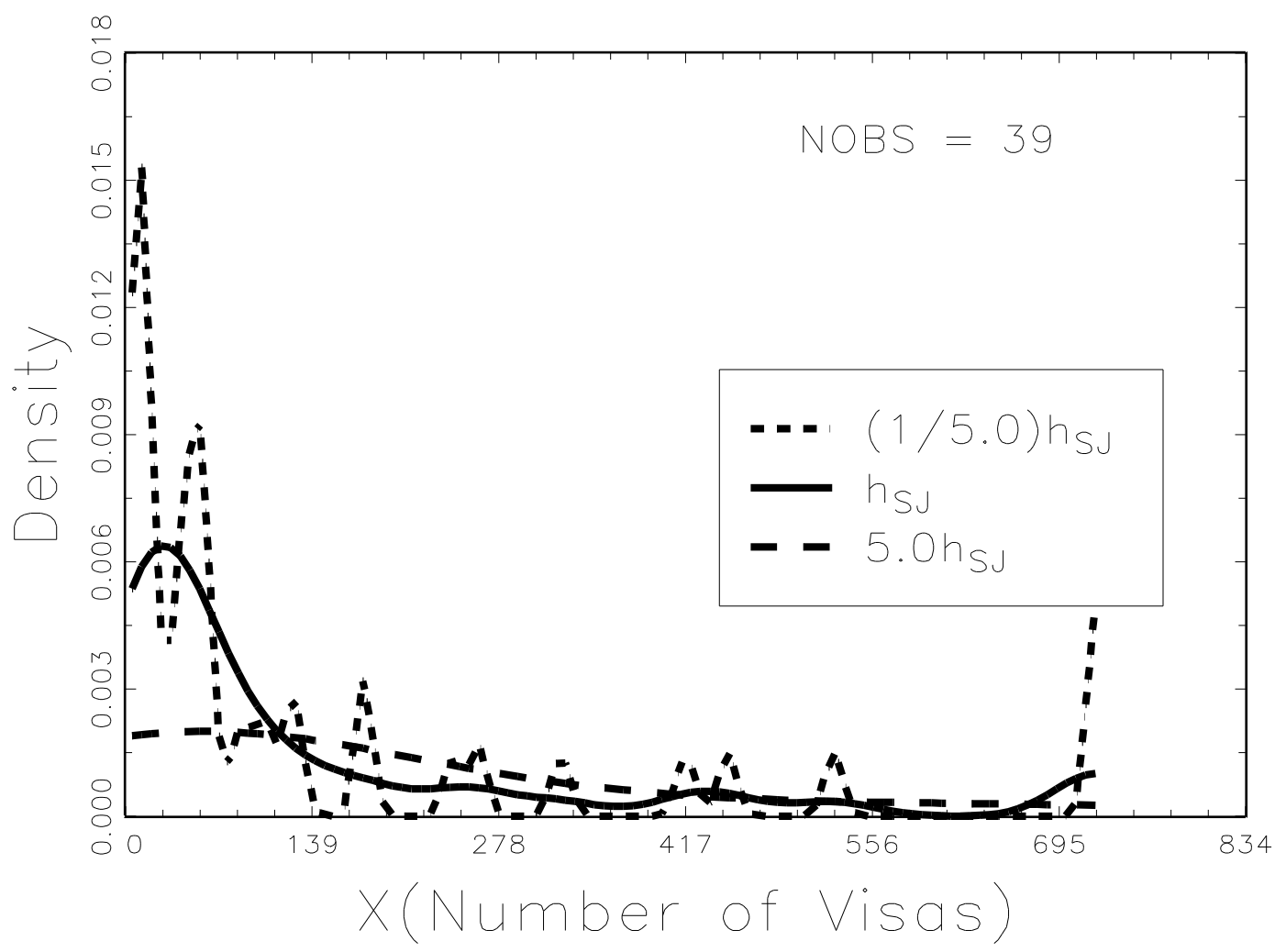

Figure 2: Transformation method estimates, the Adoption Visa data

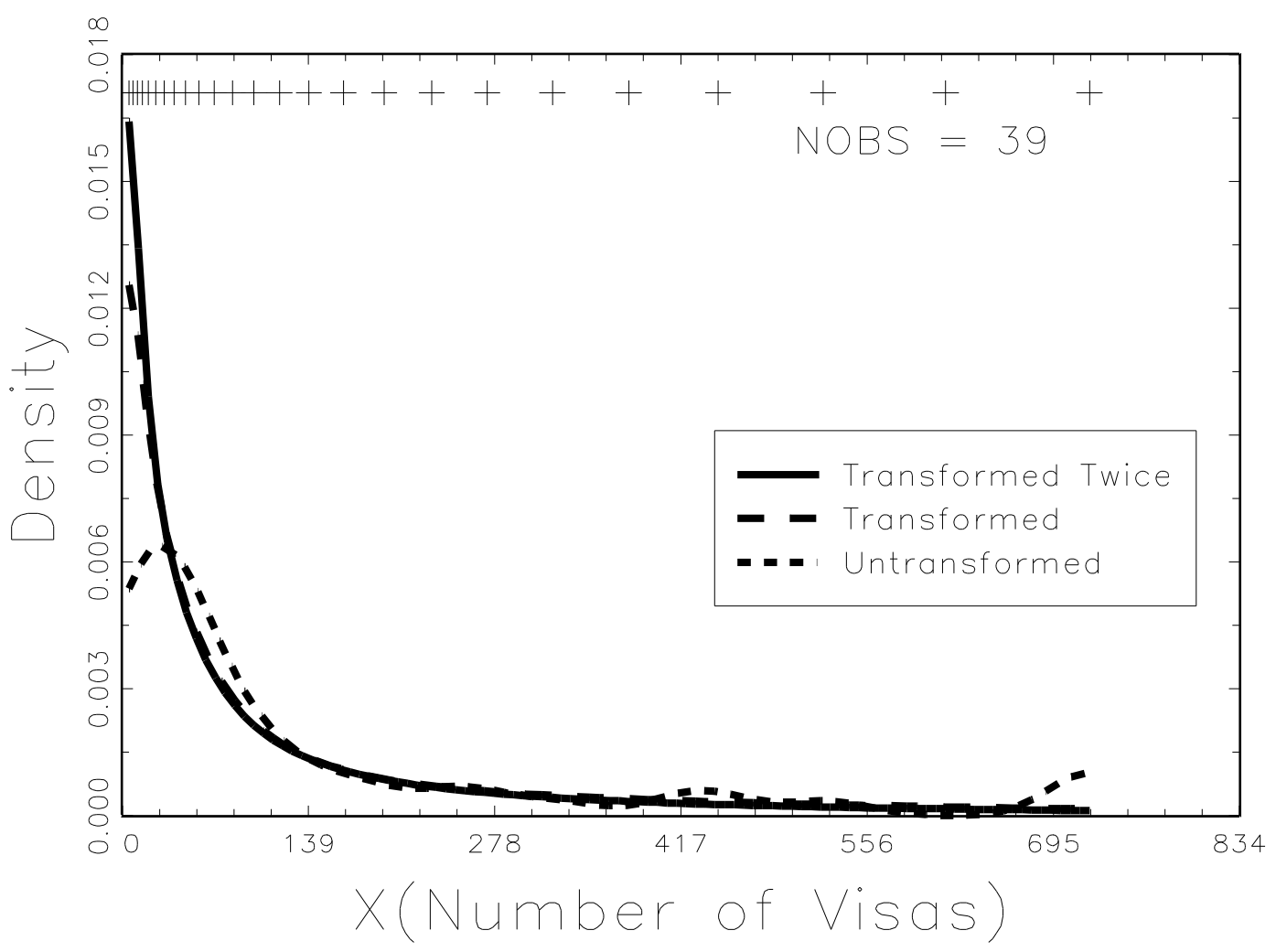


Figure 3: The effects of Johnson families

(a)

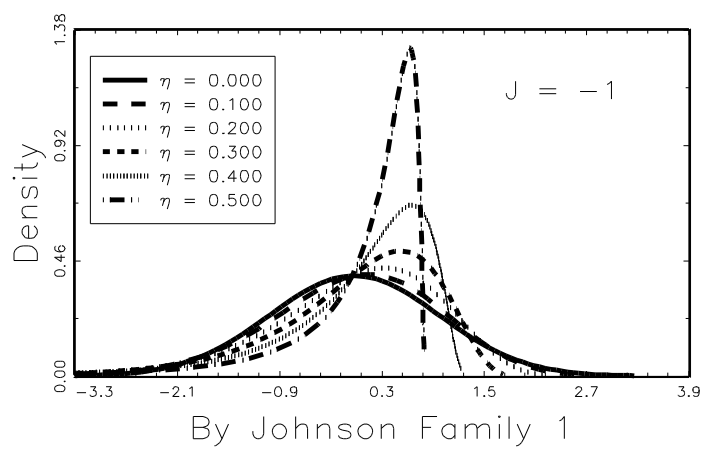

(c)

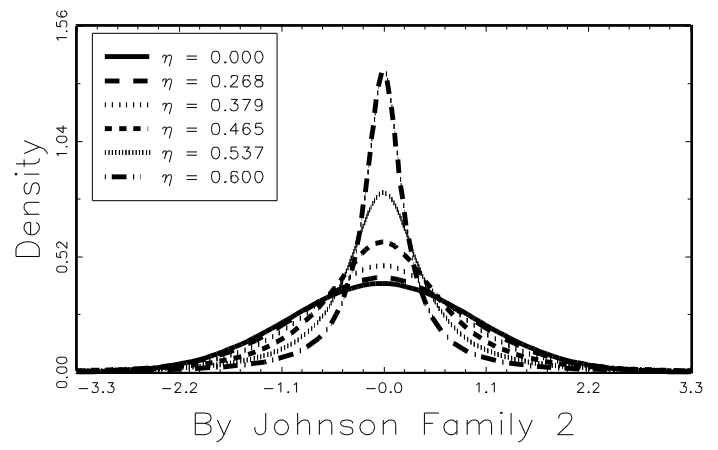

(b)

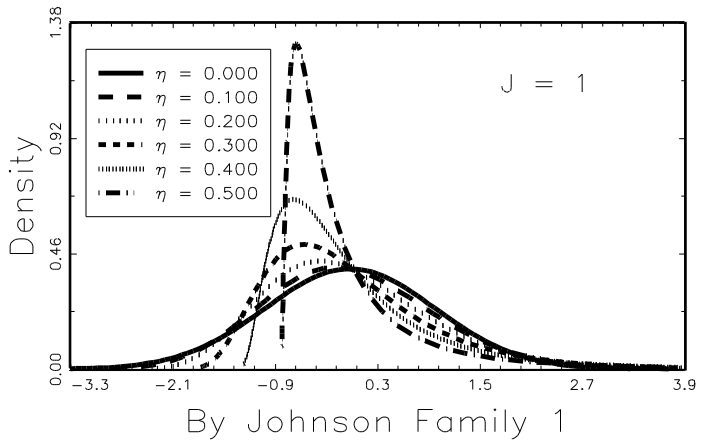

(d)

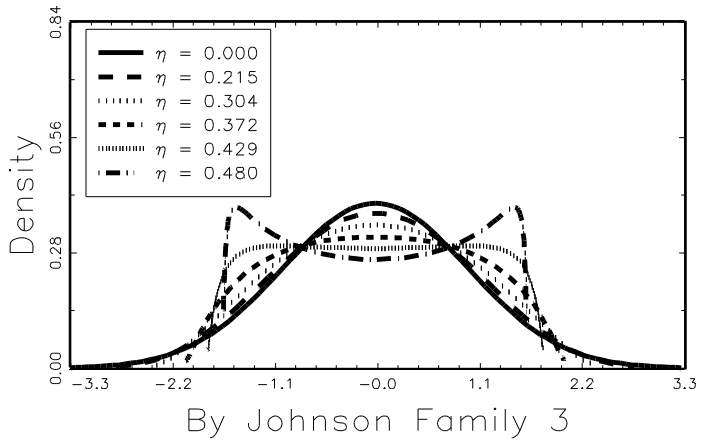

Figure 4: The Adoption Visa data, the reduction of $G(\cdot)$

(a)

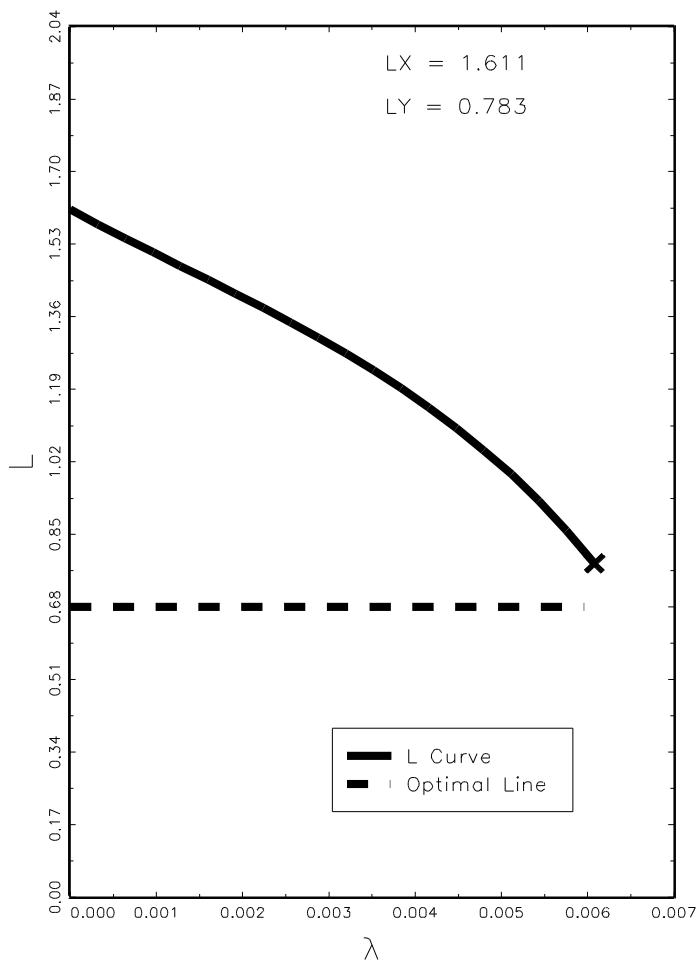

(b)

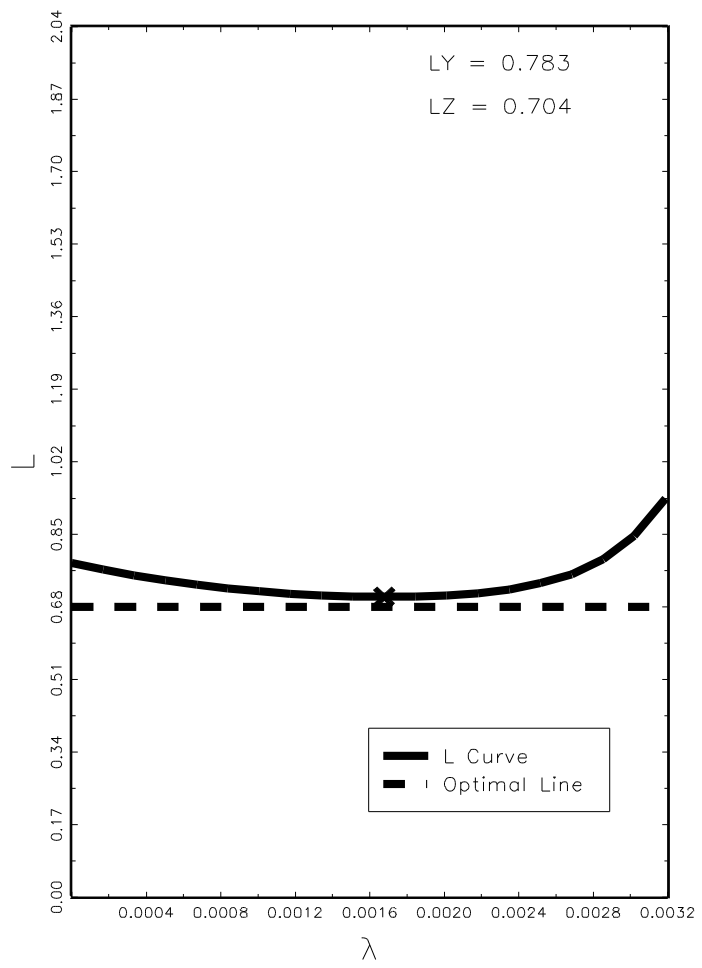


Figure 5: The Adoption Visa data, in the $Y$ and the $Z$-space

(a)

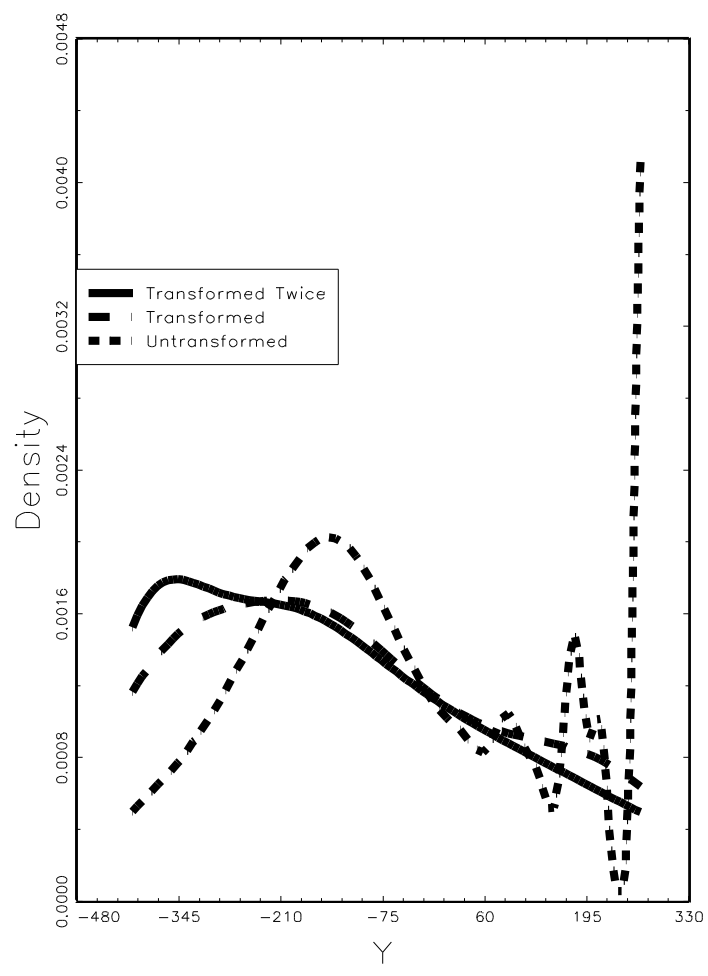

(b)

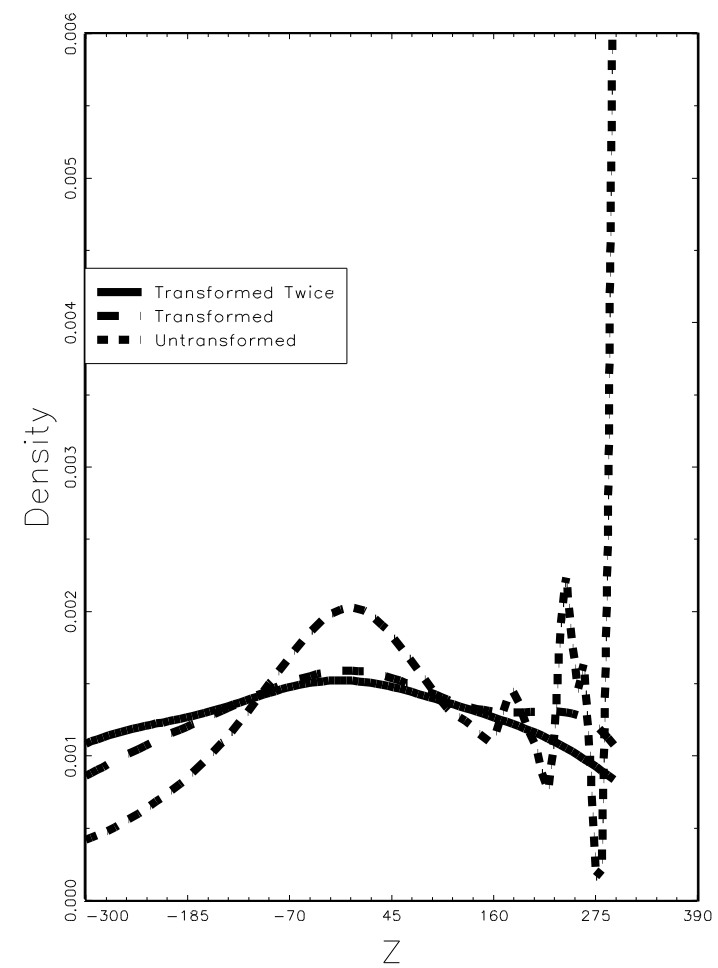

Figure 6: Transformation method estimates, the NHL Scores data

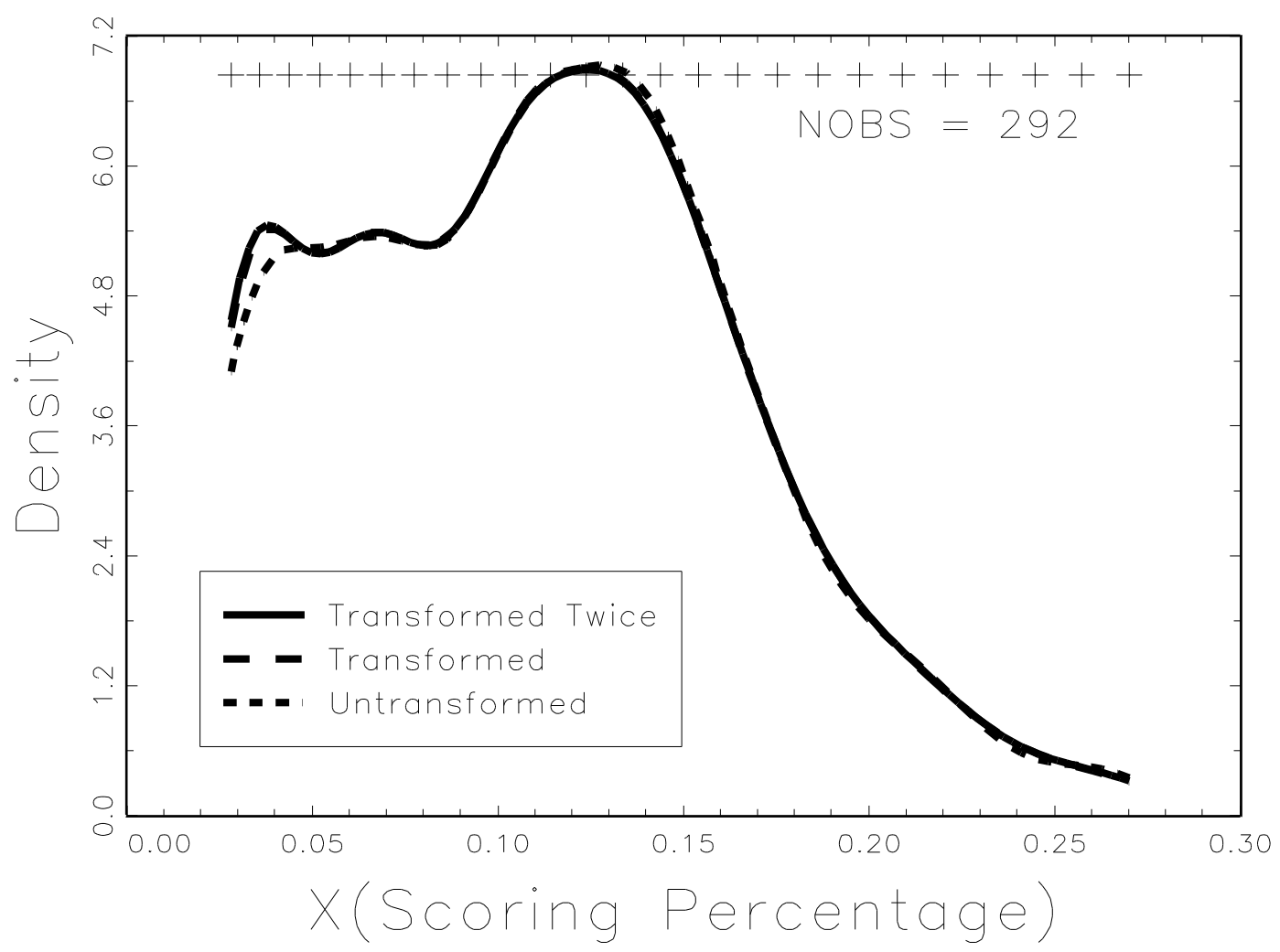


Figure 7: The Skewed M-shape density, comparison of three estimates

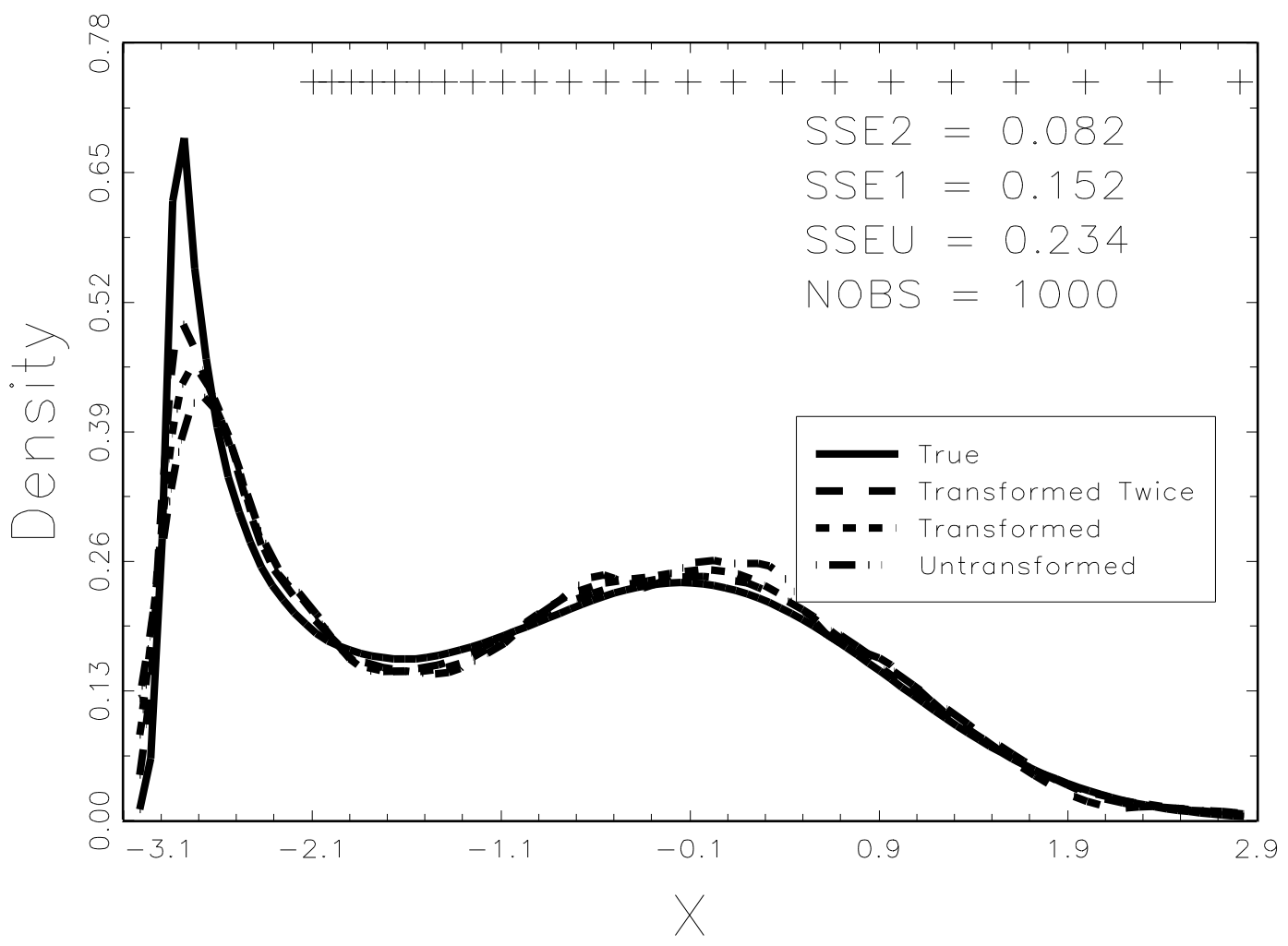

Figure 8: The Skewed M-shape density, theoretical $G(\cdot)$-values

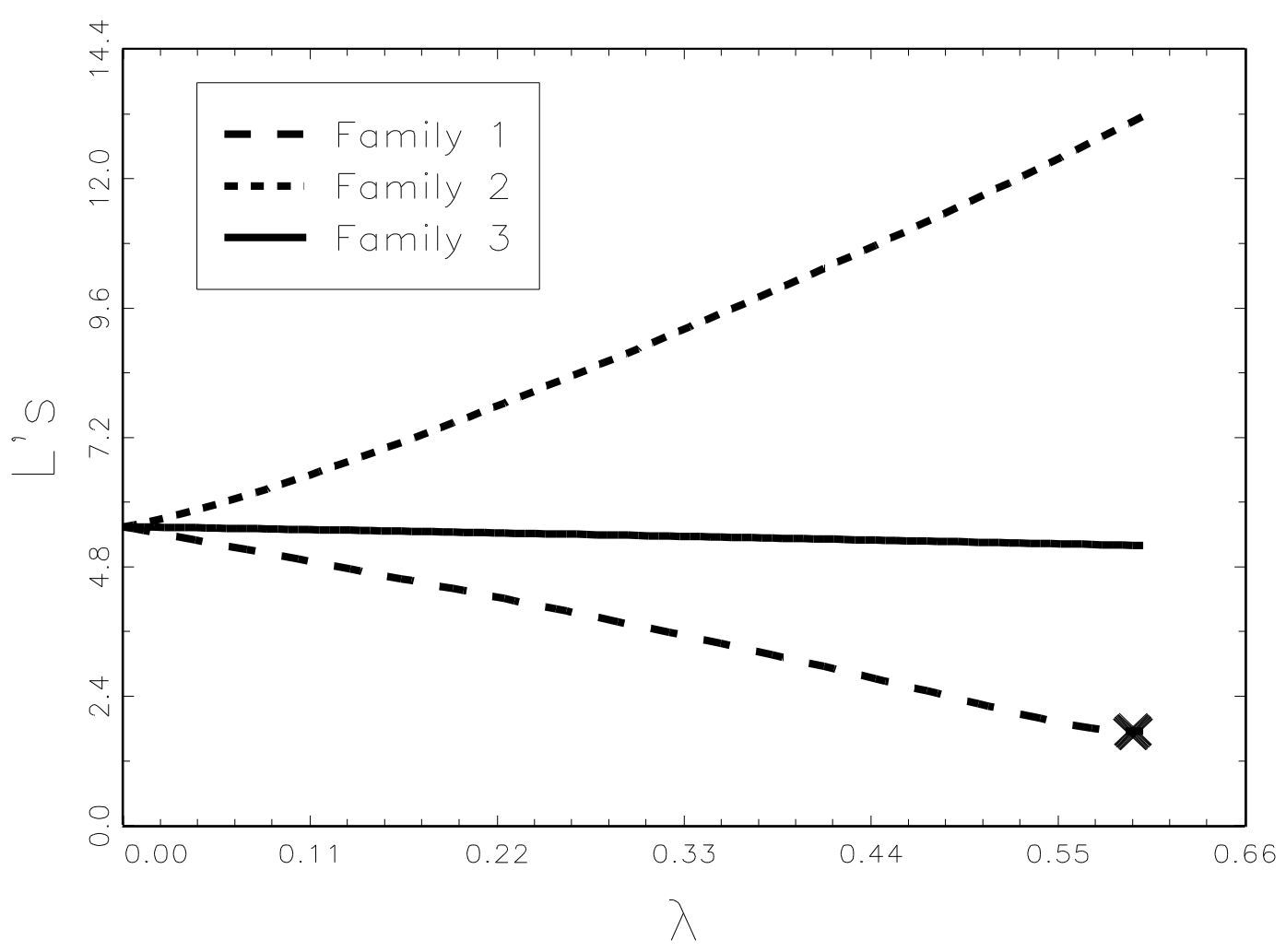


Figure 9: The Skewed M-shape density, in the $Y$ and $Z$-space

(a)

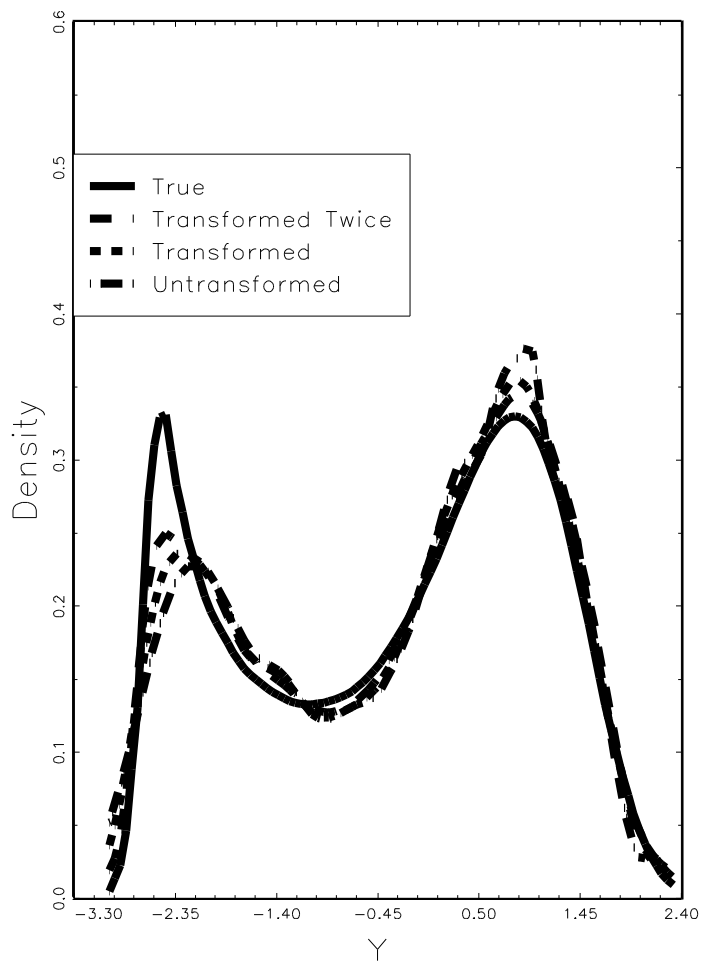

(b)

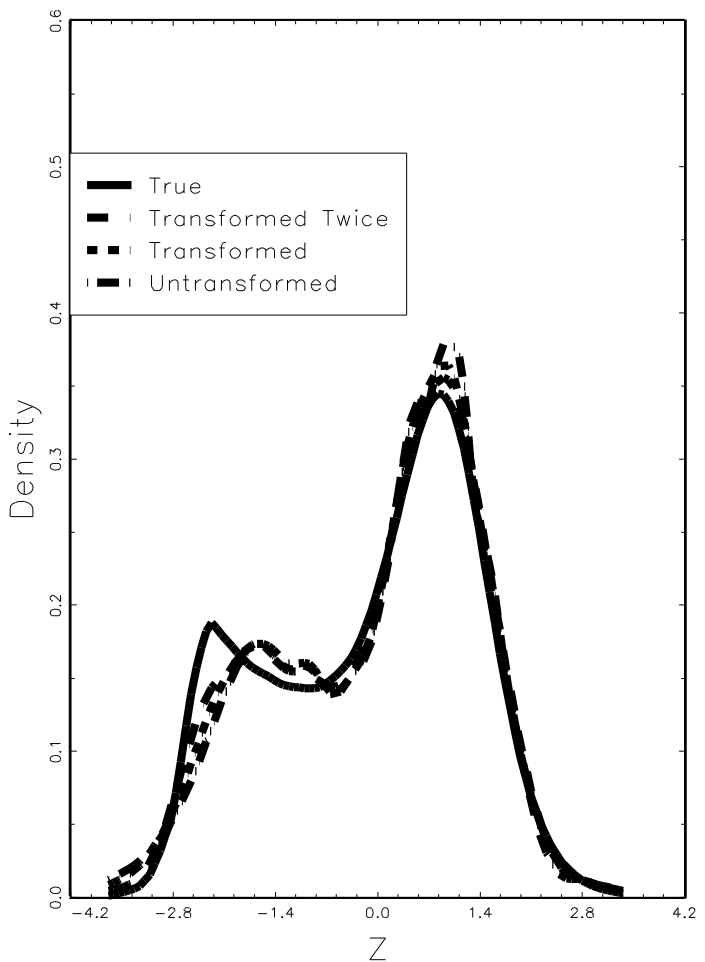

Figure 10: The Skewed M-shape density, the reduction of $G(\cdot)$

(a)

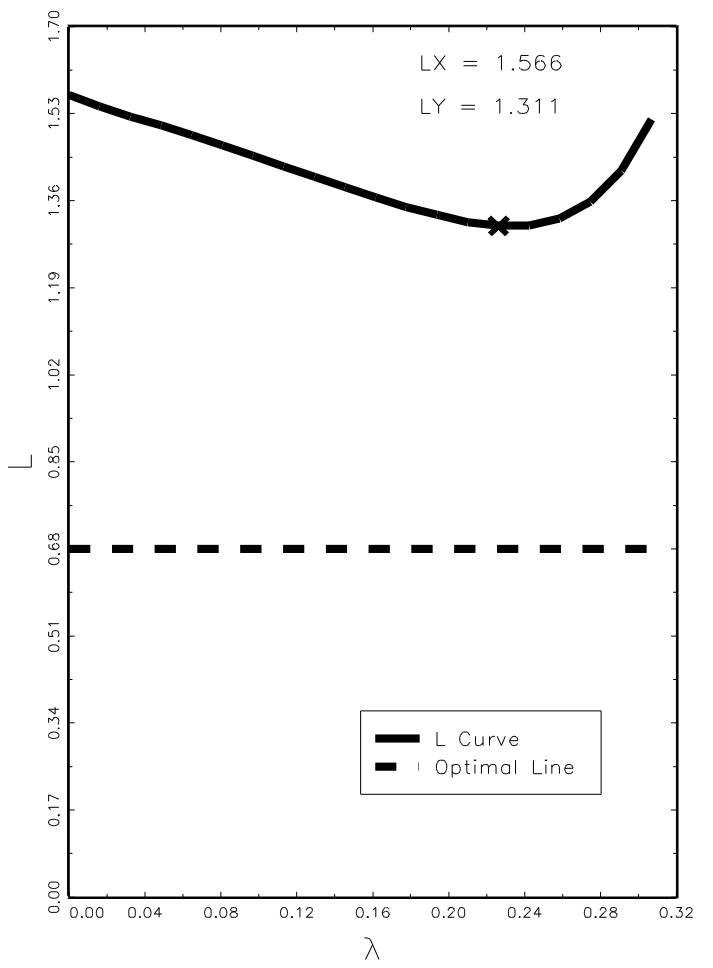

(b)

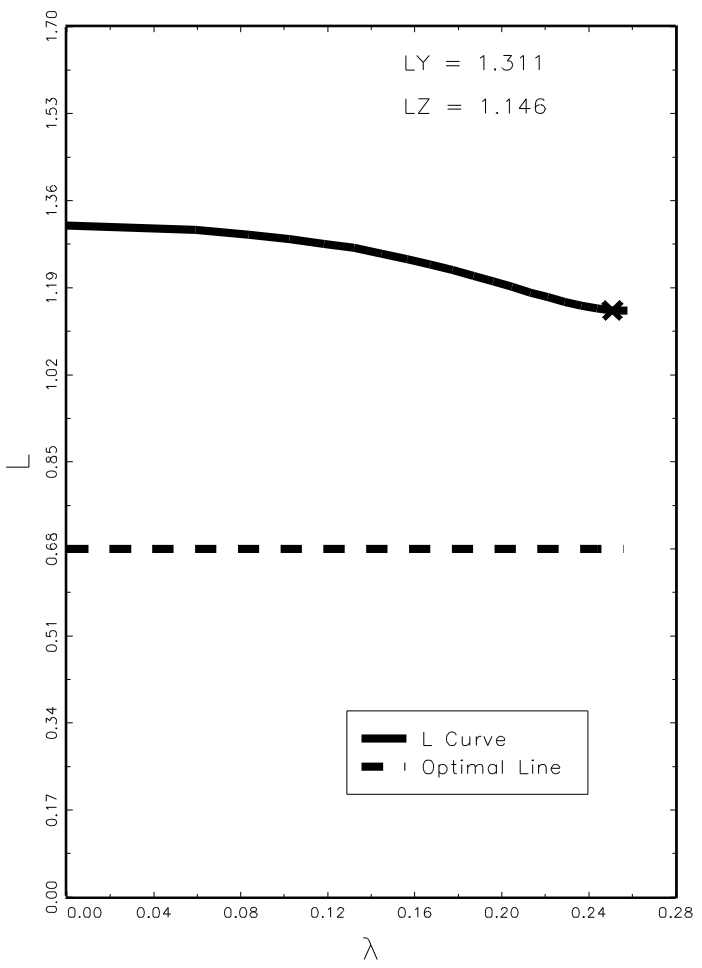


Figure 11: The Skewed M-shape density, $G(\cdot)$-values and $\log (I S E)$ 's

(a)

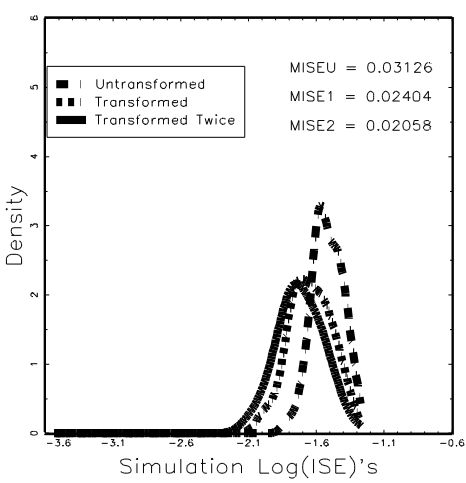

(d)

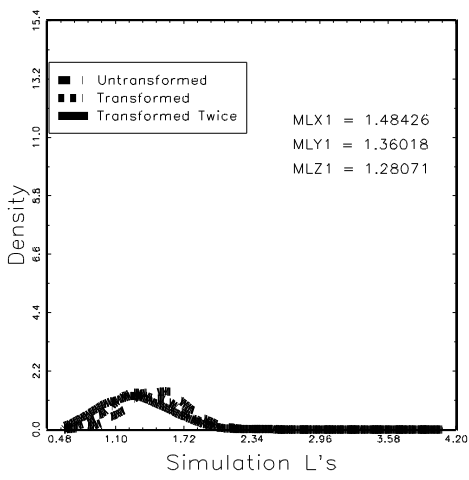

(b)

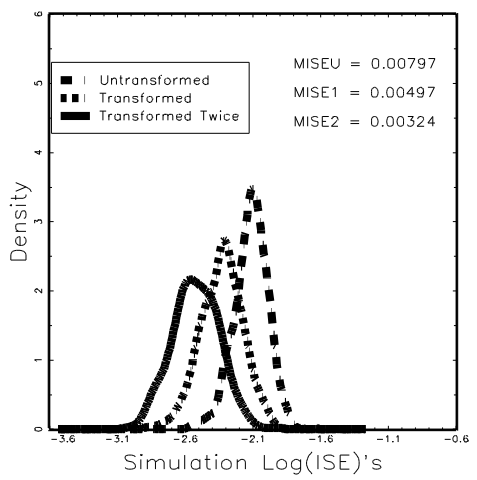

(e)

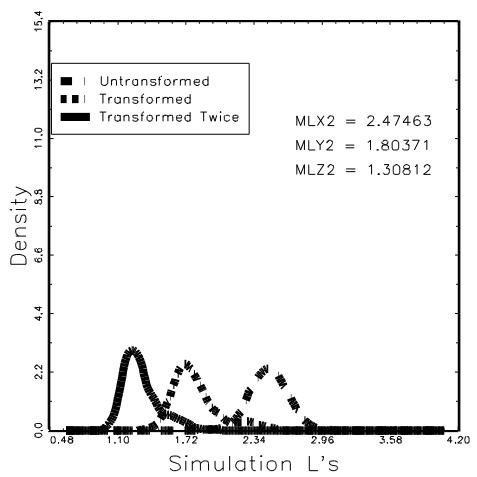

(c)

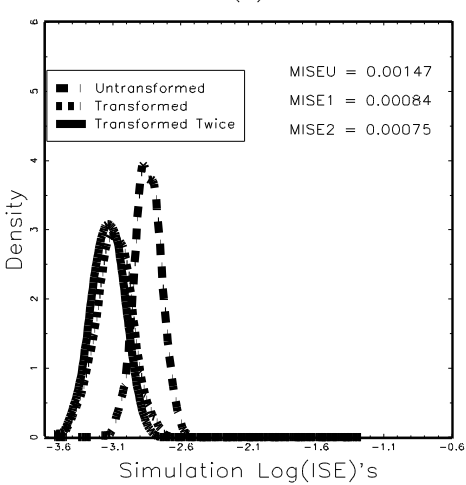

(f)

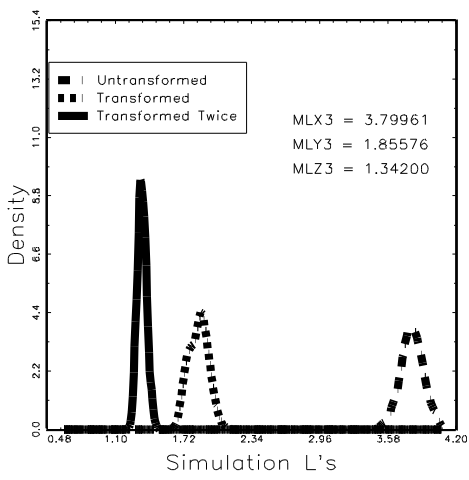

Figure 12: The Skewed M-shape density, $\hat{\lambda}$

(a)

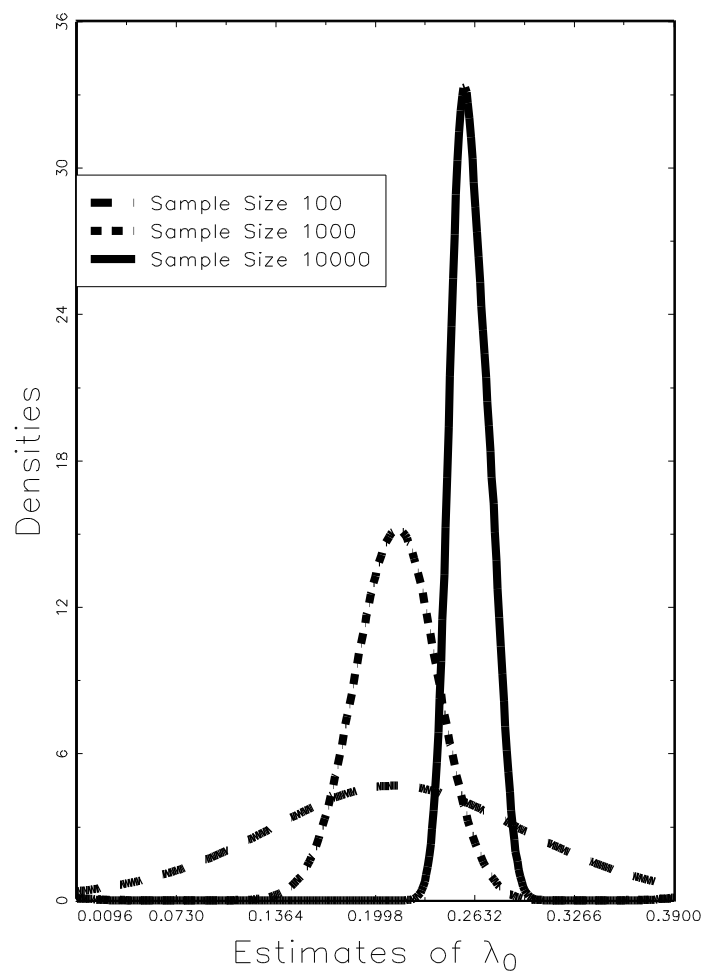

(b)

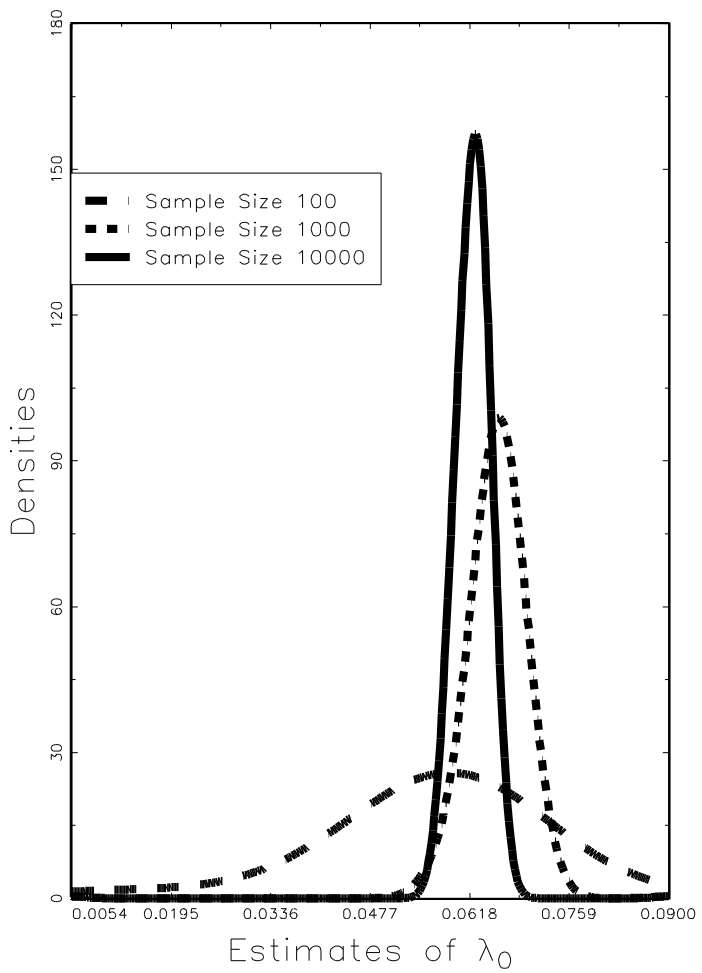

